\title{
The Intergenerational Correlation of Employment: Is There a Role for Work Culture?
}

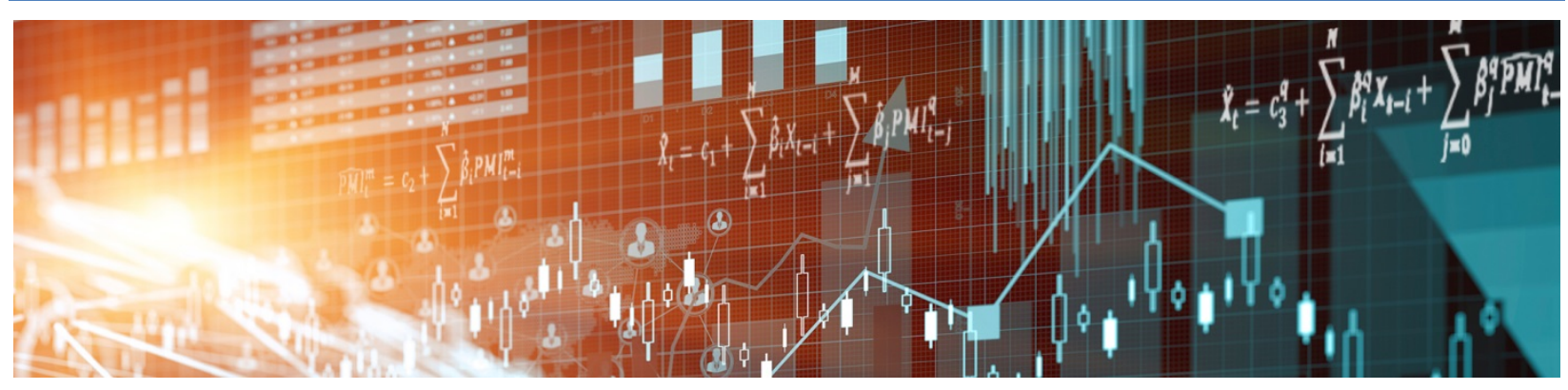

by Gabriela Galassi, David Koll and Lukas Mayr 
Bank of Canada Staff Working Paper 2019-33

September 2019

\title{
The Intergenerational Correlation of Employment: Is There a Role for Work Culture?
}

by

\author{
Gabriela Galassi ${ }^{1}$, David Koll ${ }^{2}$ and Lukas Mayr ${ }^{3}$ \\ ${ }^{1}$ Canadian Economic Analysis Department \\ Bank of Canada \\ Ottawa, Ontario, Canada K1A 0G9 \\ ggalassi@bankofcanada.ca \\ 2European University Institute \\ Department of Economics \\ Florence, Italy I-50014 \\ david.koll@eui.eu \\ 3University of Essex \\ Department of Economics \\ Colchester, United Kingdom, CO4 3SQ \\ lukas.mayr@essex.ac.uk
}




\section{Acknowledgements}

This paper has greatly benefited from discussions with professors and students at the European University Institute, Université de Montréal, UC Berkeley and Bank of Canada, as well as from presentations at the 2016 Barcelona GSE Summer Forum, the EUI Macro Working Group, and the Seminaire-midi CIREQ- Université de Montréal and the Bank of Canada Brown Bag Lunch among others. We are deeply indebted to Árpád Ábrahám, Juan

J. Dolado, Baris Kaymak, Césaire Meh, Youngmin Park, Dominik Sachs and Jan Stuhler for their fruitful comments. 


\begin{abstract}
We document a substantial positive correlation of employment status between mothers and their children in the United States, linking data from the National Longitudinal Survey of Youth 1979 (NLSY79) and the NLSY79 Children and Young Adults. After controlling for ability, education and wealth, a one-year increase in a mother's employment is associated with six weeks more employment of her child on average. The intergenerational transmission of maternal employment is stronger to daughters than to sons, and it is higher for low-educated and low-income mothers. Potential mechanisms we were able to rule out included networks, occupation-specific human capital and conditions within the local labor market. By contrast, we provide suggestive evidence for a role-model channel through which labor force participation is transmitted.

Bank topics: Labour markets; Econometric and statistical methods; Economic models JEL codes: E24, J21, J22, J62
\end{abstract}

\title{
Résumé
}

Nous montrons une corrélation positive importante entre la situation professionnelle des mères et celle de leurs enfants aux États-Unis en rapprochant les données de l'enquête National Longitudinal Survey of Youth 1979 (NLSY79) et celles de 1'enquête NLSY79 Children and Young Adults. Après avoir tenu compte des capacités individuelles, de la scolarité et de la richesse, nous trouvons que l'allongement d'un an de la durée de vie au travail de la mère est associé à une prolongation de six semaines en moyenne de la durée d'emploi de son enfant. La transmission intergénérationnelle de la durée d'emploi maternelle est plus forte chez les filles que chez les fils, et est plus forte chez les mères les moins scolarisées et à faible revenu. Parmi les possibles mécanismes de transmission, nous avons été en mesure d'exclure les réseaux, le capital humain en rapport avec l'appartenance à des catégories d'emploi particulières et les conditions du marché du travail local. En revanche, nos résultats semblent indiquer que l'exemple donné par la mère à ses enfants serait un mécanisme par lequel la participation à la population active se transmet.

Sujets : Marchés du travail, Méthodes économétriques et statistiques, Modèles économiques

JEL codes : E24, J21, J22, J62 


\section{Non-technical Summary}

For several decades, the transmission of labor market outcomes between generations has been a subject of interest. To inform about social mobility and the transmission of the earnings potential from parents to children, previous research has focused on how similar earnings of the current generation are to those of the previous one. In this paper, we focus on a different outcome, employment, as it could shed light on the transmission of willingness to work.

We use the National Longitudinal Survey of Youth 1979 (NLSY79) and the NLSY79 Children and Young Adults (CNLSY79). These allow us to link a representative set of mothers, who are born around 1960, with their children in the United States. Specifically, we document that the fraction of individuals' working-age life spent in employment is highly correlated with that of their mothers. The resulting correlation of employment is 0.21 and statistically significant; it compares with 0.4 typically documented for earnings. This means that an increase in lifetime employment of mothers by one year is associated with an increase in the employment of her child of around 11 weeks.

The correlation in employment between mothers and children remains substantial even after controlling for the main determinants of the persistence of earnings across generations (ability, education and wealth). After netting out these and some other relevant covariates, the residual correlation remains at 0.12 , corresponding to an incremental employment of children of around six weeks when maternal employment is one year higher. The result is robust to a variety of specifications and variables used to measure lifetime employment.

A different picture emerges when we consider another relevant labour market outcome, hours worked: while there is a substantial correlation of hours across generations, it does not survive after controlling for education, ability and wealth.

We further document that the residual employment correlation between mothers and their children is heterogeneous across several dimensions. First, it is significantly higher for daughters than for sons, but remains positive and statistically significant for sons as well. Second, the intergenerational correlation of employment decreases in maternal education and family income.

Finally, we consider some mechanisms that might explain the high residual intergenerational correlation. First, our results show that for mothers and children sharing the same industries, occupations and local labor markets correlations are not higher. This rules out network effects, occupation-specific human capital or local labor markets as alternative explanations for the residual correlation. Second, the residual correlation also remains after controlling either for work preferences of the mother or their work behavior when the child is not living with her. It seems important that the child observes her/his mother's working behavior, favoring a role model channel to be the driving force of the transmission of employment status across generations.

Overall, the positive and strong intergenerational correlation of employment, which is to the best of our knowledge a novel fact, cannot be explained by human capital, wealth or direct preference transmission and has important implications not only for the analysis of social mobility but, potentially, also for the optimal design of tax-transfer policies. 


\section{Introduction}

For several decades, the intergenerational correlation of labor market outcomes has been a subject of interest among both academics and policy-makers. As a key determinant of socioeconomic mobility, the correlation of labor earnings between subsequent generations has received particular attention. An extensive literature documents that earnings of individuals are highly correlated with those of their parents (see the comprehensive surveys by Solon, 1999; Bowles and Gintis, 2002; Black and Devereux, 2011; Björklund and Jäntti, 2011). The focus of this literature is on the identification and quantification of channels through which the "potential" to earn is transmitted. Such channels include, among others, the genetic inheritance of cognitive skills, higher investments into children's education by parents with higher income, and parents' social networks, which children can take advantage of.

By contrast, in this paper we argue that not only "earnings potential" is transmitted across generations but also the "willingness to work". Specifically, we document that employment status, or the fraction of individuals' working-age life spent in employment, is highly correlated with their mothers'. This correlation remains significant after controlling for the main determinants of the intergenerational correlation of earnings, according to the literature on the topic. The positive and strong intergenerational correlation of employment - a novel fact, to the best of our knowledge - has important implications not only for the analysis of social mobility but, potentially, also for the optimal design of tax-transfer policies.

Why has this fact been overlooked so far? Perhaps the reason is that the empirical literature on earnings correlations typically restricts the analyzed sample to individuals and periods for which earnings are observed, thereby neglecting the variation in employment status (i.e. the extensive margin of labor supply) by construction. Such data, of course, still capture some variation in labor supply, namely the variation in hours worked, or the intensive margin of labor supply. However, our evidence shows that while the unconditional intergenerational correlations of both employment and hours are substantial, only the one for employment remains significantly different from zero after controlling for education, ability, and wealth. This means, on the one hand, that employed individuals tend to work a similar number of hours to their mothers simply because their benefits from work (wages determined to a large extent by ability and education) as well as their need to work (determined by wealth) are similar. On the other hand, it also means that these same factors are not able to fully explain the high correlation in the decision on whether to work at all.

We obtain our results by linking data from the National Longitudinal Survey of Youth 
1979 (NLSY79) and the Children and Young Adults (CNLSY79) cohort. These data are designed to link mothers from a representative sample born in the US between 1957 and 1964 with their children. Since there is more variation in the female labor supply than in the male labor supply, we believe the focus on mother-children pairs is reasonable given our goal. Exploiting the longitudinal structure of the data, we first estimate the permanent component of employment status along the life cycle for both, mothers and children. This permanent component measures how much of their active life individuals spend in employment. The information included in this component is different from the permanent component of earnings, which is based only on periods of employment when earnings are observed.

We find a robust, statistically significant and positive correlation of employment status $1^{1}$ The unconditional correlation is 0.21 , implying that an increase in lifetime employment of mothers by one year is associated with an increase in the employment of her child of around 11 weeks. After netting out the influence of ability, education, wealth, and some other relevant covariates, the correlation remains at 0.12 , corresponding to an incremental employment of children of around six weeks. This is what we call residual correlation of employment:2

Furthermore, by splitting the sample into different sub-samples, we find that the residual employment correlation between mothers and their children is heterogeneous across several dimensions. First, it is significantly higher for daughters (0.18) than for sons (0.07). While a one-year increase in lifetime employment of mothers increases the employment of their sons by on average less than four weeks (still significant at the $5 \%$ level), it increases employment of their daughters by more than nine weeks. This result contrasts with the literature analyzing gender differences in the intergenerational earnings elasticity, which has documented lower elasticities for daughters with respect to their parental income (see, for example, Chadwick and Solon, 2002; Olivetti and Paserman, 2015). Second, the intergenerational correlation of employment is decreasing in the degree of maternal education, being significantly positive only for mothers without any college education. Finally, the correlation is highest for the bottom quintile of the income distribution and tends to decrease in income.

The significant intergenerational correlation of the extensive - rather than the intensive margin of labor supply is particularly interesting in light of several existing policies, such as the Earned Income Tax Credit (EITC) in the United States, which aim to encourage labor force participation. This is especially the case since the correlation is higher at the bottom

\footnotetext{
${ }^{1}$ In the Appendix we provide results for an extensive set of different specifications, all of which confirm our main result.

${ }^{2}$ To put these numbers into perspective, estimates for the intergenerational earnings elasticity in the US have oscillated around 0.4 (see, for example, Solon, 1992, Zimmerman, 1992, Chetty et al., 2014).
} 
of the income distribution, the target group of the EITC. Our results suggest that there may be a dynamic fiscal benefit of such policies through increased labor market participation, and hence tax revenue, of future generations.

However, before such conclusions can be drawn, an understanding of the channels determining this correlation is needed. Two pieces of evidence suggest the existence of a role-model effect. First, as mentioned above, the correlation of employment status is higher for mother-daughter pairs than for mother-son pairs, and role models tend to be more pronounced within the same gender. Second, we use certain survey questions in the NLSY79 to extract a measure for work preferences of mothers. This measure is significantly correlated with maternal employment but uncorrelated with children's employment, suggesting that it is not innate preferences for work that are transmitted across generations. Instead, it seems important that the child observes her/his mother working.

Finally, we rule out alternative explanations for this residual correlation, such as the effect of networks, occupation-specific human capital, or local labor markets. Particularly, we analyze the heterogeneity in the intergenerational correlation of employment across motherchildren pairs that do or do not share industries, occupations, or local labor markets. The lack of difference across groups indicates that these explanations are unlikely to drive the intergenerational correlation of employment status.

Related literature. Our paper contributes to many different branches of the empirical literature studying the transmission of preferences for work across generations. Using tools of the well-established literature on the intergenerational correlations of labor market outcomes $3^{3}$ we focus on an unexplored variable, employment, and argue that it bears important information on transmission of preferences for work.

The gender literature has analyzed the transmission of preferences for work from the perspective of gender roles. An important part of this literature uses the so-called "epidemiological approach". This approach considers the intergenerational transmission of cultural traits when outcomes of second-generation migrants and those of the parents' country of origin are correlated. Fernandez (2007) and Fernandez and Fogli (2009) interpret such correlation in female labor force participation as cultural transmission of women's roles. Another, more structural, strand of the gender literature also looks at cultural transmission. For instance, Fernandez (2013) explains the S-shape in the female labor force participation during

\footnotetext{
${ }^{3}$ The literature dealing with methodological issues for measuring intergenerational correlations in reduced form, on which we rely, is vast (see, for example, Solon, 1992, 1999; Haider and Solon, 2006; Grawe, 2006 Lee and Solon, 2009; Nybom and Stuhler, 2016, 2017| Mazumder.|2005).
} 
the second half of the $20^{\text {th }}$ century with a model that introduces learning across generations about the returns to female work.

These studies deal with the transmission of society-wide preferences. We instead analyze preference transmission within the family, from mothers to children. Furthermore, our paper does not limit attention to the transmission of gender roles, as we do not restrict the analysis to mothers and daughters. In this last sense, our paper distances itself from others that have analyzed the transmission of gender roles (see, for example, Binder, 2018; Olivetti et al., 2018).

Another related strand of literature documents that parental welfare benefit reception results in an increased probability of children claiming the benefits themselves (see, for example, Dahl, Kostøl and Mogstad, 2014; Dahl and Gielen, 2018; Hartley, Lamarche and Ziliak, 2017). Even though the results in this literature are consistent with a transmission of work preferences, they could also be explained by other factors such as the transmission of stigma or information about welfare benefits 4 Furthermore, dependence on welfare benefits is not necessarily the complement of employment, our outcome of interest. Non-employed individuals may receive other sources of non-labor income for their support, such as family transfers, and not resort to welfare benefits.

The studies most closely related to this paper are those that infer transmission of work preferences from observed labor market outcomes. Estimating an overlapping generations model with data from the Panel Study of Income Dynamics, Toledo (2010) attributes the correlation in hours worked between fathers and sons to the transmission of preferences for work. Altonji and Dunn (2000) reach a similar conclusion using the National Longitudinal Survey of Labor Market Experience and relying on a factor model that allows preferences to influence labor market outcomes. Both Toledo (2010) and Altonji and Dunn (2000) find that transmission of preferences for leisure or work are the main drivers of intergenerational correlations in hours worked, while productivity or wages explain the earnings correlations. The focus on hours worked, or the intensive margin of labor supply, is the main difference to our paper 5 As discussed above, this distinction is important, as only the correlation of employment remains significant after controlling for education, ability, and wealth. This in

\footnotetext{
${ }^{4}$ In a related paper, Dahl, Loken and Mogstad (2014) document important peer effects within the workplace and the family magnifying social programs' participation, favoring an interpretation of information transmission.

${ }^{5}$ Another difference with both Toledo (2010) and Altonji and Dunn (2000) is that our data allow to control for ability and human capital. They instead use observed wages, which are endogenous to the labor supply decision.
} 
turn suggests that the proposed role-model effect works through the extensive rather than the intensive margin of the mother's labor supply.

Outline. The remainder of the paper is structured as follows. In Section 2, we present the data, followed by the empirical strategy in Section 3 . Section 4 documents the main results. In Section 5, we discuss potential mechanisms. Section 6 concludes.

\section{Data}

We use the National Longitudinal Survey of Youth 1979 cohort (NLSY79) and the Children and Young Adults cohort (CNLSY79). These data are widely used in the analysis of inequality and labor market research. The NLSY79 surveys a representative sample of individuals born in the US between 1957 and 1964. Respondents are 14 to 22 years old in 1979 and are followed since then. Our last observation is 2012, when they are 47 to 56 years old. The frequency is annual between 1979 and 1994, and biannual thereafter. The children of the women in this cohort are surveyed on a biannual basis since 1986, constituting the CNLSY79. They are linked to the original cohort by a unique identifier provided by the US Bureau of Census 6

We restrict the analysis to the cross-sectional sub-sample of the NLSY79 that is designed as a representative sample of the US population in 1979. We exclude other sub-samples that oversample particular groups of the population, to avoid weighting the estimates. We restrict to observations during ages 25 to 45 for both cohorts to keep the representativeness of the lifetime employment experience (the oldest individual in the second cohort is 38 years old in 2012). We obtain a final sub-sample of 1,373 mothers paired to 2,339 children.

The data are particularly rich. They provide detailed information on labor market outcomes, education, and further demographic and socio-economic characteristics. Importantly, they contain widely used indicators of ability, which is a key confounder for the estimation of intergenerational transmission of labor market outcomes: the Armed Forces Qualification Test (AFQT) for the mothers and the Peabody Individual Achievement Test (PIAT) for the children; we use the Math score of the latest PIAT assessment for the children cohort, in line with the literature (Abbott et al., 2013). We use information on wealth (net worth), com-

\footnotetext{
${ }^{6}$ Although in the NLSY79, only mothers (and not fathers) can be linked to CNLSY79 data, this does not challenge the objective of our paper. As we focus on the extensive margin of the labor supply decision, using maternal employment information is reasonable because female labor force participation is typically lower (through more elastic labor supply) than male labor force participation, particularly during the period of observation of the first cohort.
} 
puted as assets (savings, home and vehicle ownership) minus debts (credit cards, students loans, mortgages, vehicle loans, and others).

Table 1 provides descriptive statistics of the data (additional descriptives are summarized in Table C.8 in the Appendix). For most variables, we report the means across individual averages for those observations over the 25 to 45 years old range in our sample. The last two columns refer to the sample of mothers and their children, and the first one shows the characteristics of the total sample of women in the NLSY79 cohort for reference. All monetary values are deflated using the Consumer Price Index (CPI) and expressed in prices of 1980 .

Mothers are observed on average for 14 waves, and children for 2.5 waves. The average age is 33 for mothers and 27 for children. The sample of mothers is representative of women with children by design. As compared with the total sample of women in the NLSY79, mothers are slightly less educated and live in poorer households. Women are 22 years old on average when they give birth. The children's cohort is relatively younger than the mothers' by construction, as reflected in the age and other characteristics associated to the life cycle (for example, the proportion married and cohabiting is lower in the children's cohort, and the wealth level as well). Observations of older children correspond to younger mothers at the time of birth. Children are slightly more educated than mothers.

Questions about employment status vary across waves in the survey. Our choice of the particular question used in our analysis balances two objectives: (i) we want to have a measure that is as homogeneous as possible between the samples of mothers and children; (ii) at the same time, the questions should be consistent along the different waves and minimize the number of non-responses. We consider mothers to be employed if they declare that they worked for 10 or more weeks in the year before the interview. We categorize children as employed if their earnings in the year before the interview were equivalent to at least two months of a part-time job at the minimum salary 7 The employment rate is $73 \%$ for mothers and $84 \%$ for the children cohort ( $80 \%$ for daughters). Although these figures seem high as compared with official statistics of female employment, they are not at odds, considering that we are taking an annual window for the measurement of employment.

Employed mothers and children work on average 36 and 40 hours a week at an hourly wage rate of $\$ 7$ and $\$ 6$ (in 1980 USD), respectively. Earnings amount to $\$ 9,750$ and $\$ 13,316$

\footnotetext{
${ }^{7}$ The lower bound for earnings is arbitrary, although reasonable. It is $\$ 450$ monthly in 1980 USD, which is equivalent to a job of 17 hours a week (part-time), for 8.5 weeks (2 months) and $\$ 3.1$ per hour. The main purpose is to exclude casual jobs. We also show that the results are robust to other measures of employment.
} 
Table 1: Summary statistics for women and mother-child pairs in NLSY79 and CNLSY79

\begin{tabular}{|c|c|c|c|}
\hline & Women & Mothers & Children \\
\hline \multicolumn{4}{|l|}{ Demographics } \\
\hline Age & $\begin{array}{l}32.9 \\
(1.7)\end{array}$ & $\begin{array}{c}33.2 \\
(1.0)\end{array}$ & $\begin{array}{l}27.3 \\
(1.6)\end{array}$ \\
\hline Female & $100 \%$ & $100 \%$ & $50 \%$ \\
\hline Married/cohabiting & $67 \%$ & $77 \%$ & $33 \%$ \\
\hline Number of children & $\begin{array}{c}1.9 \\
(1.4)\end{array}$ & $\begin{array}{c}2.6 \\
(1.2)\end{array}$ & $\begin{array}{c}1.2 \\
(1.3)\end{array}$ \\
\hline Maternal age at birth & & & $\begin{array}{l}21.7 \\
(3.4) \\
\end{array}$ \\
\hline \multicolumn{4}{|l|}{ Education and Ability } \\
\hline Years of education & $\begin{array}{l}13.7 \\
(2.6)\end{array}$ & $\begin{array}{l}12.8 \\
(2.2)\end{array}$ & $\begin{array}{l}13.4 \\
(2.4)\end{array}$ \\
\hline High school drop-out & $7 \%$ & $10 \%$ & $12 \%$ \\
\hline High school complete & $40 \%$ & $51 \%$ & $36 \%$ \\
\hline Some college & $25 \%$ & $26 \%$ & $28 \%$ \\
\hline Complete college & $28 \%$ & $13 \%$ & $24 \%$ \\
\hline Percentile in cognitive test & $\begin{array}{c}48.8 \\
(28.5)\end{array}$ & $\begin{array}{c}39.6 \\
(26.9)\end{array}$ & $\begin{array}{c}49.1 \\
(27.8)\end{array}$ \\
\hline Age at test & $\begin{array}{l}18.0 \\
(4.0)\end{array}$ & $\begin{array}{l}18.3 \\
(4.2)\end{array}$ & $\begin{array}{l}11.6 \\
(4.6)\end{array}$ \\
\hline \multicolumn{4}{|l|}{ Labor Market Outcomes } \\
\hline Employment & $79 \%$ & $73 \%$ & $84 \%$ \\
\hline Hours/week & $\begin{array}{l}37.8 \\
(8.3)\end{array}$ & $\begin{array}{l}36.4 \\
(8.8)\end{array}$ & $\begin{array}{c}39.6 \\
(11.0)\end{array}$ \\
\hline Hourly wage (in USD) & $\begin{array}{c}8.1 \\
(8.2)\end{array}$ & $\begin{array}{c}6.7 \\
(9.9)\end{array}$ & $\begin{array}{c}6.3 \\
(4.0)\end{array}$ \\
\hline Annual earnings (in 1,000 USD) & $\begin{array}{l}12.9 \\
(9.1)\end{array}$ & $\begin{array}{c}9.8 \\
(6.7) \\
\end{array}$ & $\begin{array}{l}13.3 \\
(9.8)\end{array}$ \\
\hline \multicolumn{4}{|l|}{ Wealth and Income } \\
\hline Net worth (in 1,000 USD) & $\begin{array}{c}55.9 \\
(95.9)\end{array}$ & $\begin{array}{c}43.1 \\
(81.6)\end{array}$ & $\begin{array}{c}9.6 \\
(31,2)\end{array}$ \\
\hline Family income (in 1,000 USD) & $\begin{array}{c}33.5 \\
(35.6)\end{array}$ & $\begin{array}{c}27.2 \\
(24.5)\end{array}$ & $\begin{array}{c}26.0 \\
(29.1)\end{array}$ \\
\hline Number of interviews & $\begin{array}{l}13.2 \\
(3.1)\end{array}$ & $\begin{array}{l}14.1 \\
(1.9)\end{array}$ & $\begin{array}{c}2.5 \\
(1.2)\end{array}$ \\
\hline Individuals & 3,040 & 1,373 & 2,339 \\
\hline
\end{tabular}

Notes: Averages for quantitative variables (standard deviations in parentheses), percentages for dichotomous variables, for observations in the 25 to 45 years old range in our sample. Cognitive tests are AFQT for parents and PIAT Math for children. Monetary variables are in 1980 USD. 
annually. Net worth is higher for the mothers' than for the children's cohort $(\$ 43,064$ vs. $\$ 9,551)$, a difference potentially due to the composition of the children's sample explained above, as well as because most children had not inherited yet at the time they were surveyed. No such differences are observed in family income across cohorts, though $(\$ 27,226$ and $\$ 26,029$, respectively). The average percentile of maternal cognitive test scores is 40 , and it is 49 for children. Mothers take the test when they are 18 years old and children when they are 12. Further details on the data can be found in Appendix B.1.

\section{Empirical strategy}

We follow the literature on intergenerational correlations of labor market outcomes to quantify the persistence in employment status across generations. The unit of observation is the mother-child pair $i$ and our main regression specification relates the permanent component of employment - which can be interpreted as the fraction of lifetime employment - of the mother $l_{M i}$ to the permanent component of employment of the child $l_{C i}$. The reduced-form specification is

$$
l_{C i}=\alpha+\beta l_{M i}+\phi_{M} X_{M i}+\phi_{C} X_{C i}+\epsilon_{i} .
$$

Our coefficient of interest, $\beta$, summarizes the intergenerational persistence of employment. $X_{M i}$ and $X_{C i}$ are control variables for mothers and children, respectively. We consider different specifications and control for several confounders, including education, ability, wealth, the number of children of both generations, and the age of the mother at birth.

Computation of permanent components. Equation (1) relies on measures of lifetime employment status. The literature on intergenerational correlations is quite rich in terms of how to compute these lifetime or long-run measures. Given the nature of our data, we take an approach that allows for the use of all the periods of information. Following Zimmerman (1992) and Toledo (2010), we obtain these lifetime or permanent components of employment as the fixed effects in a statistical model for the probability of being employed in each period under observation 8

\footnotetext{
${ }^{8}$ Using multiple periods has been shown to reduce measurement error (see, for example, Solon, 1992 Mazumder, 2005, Haider and Solon, 2006). This strategy is simpler than a factor model that explicitly models such error (see, for example, Lochner et al., 2018), but we consider it effective, particularly for employment, the main focus of this paper. Lee and Solon (2009) recommend an efficient approach by using all the children's observations in a version of the intergenerational equation (1). Our approach also uses all the information of the children, but in a two-step procedure that we deem accurate according to the Frisch-Waugh-Lovell theorem.
} 
We specify a linear probability model,

$$
l_{k i t}=l_{k i}+\sum_{n=1}^{2} \pi_{n k} A_{k i t}^{n}+\lambda_{k t}+v_{k i t}
$$

which we run for both generations $k \in\{M, C\}$. Specifically, we assume that the probability of individual $i$ to be employed in year $t$ is a function of a second-order polynomial of the individual's age $A_{k i t}$, a year fixed effect $\lambda_{k t}$, and an individual fixed effect $l_{k i}$. This individual fixed effect represents the permanent component of employment status, abstracting from lifecycle fluctuations (absorbed by age effects), and from business-cycle fluctuations (absorbed by year effects). We can interpret the permanent component of employment as the proportion of lifetime each individual is in employment.

Regression versus correlation coefficient. An alternative to the regression coefficient $\beta$ for measuring persistence in labor market outcomes across generations is the correlation coefficient 9

$$
\rho=\beta \frac{\sigma_{M}}{\sigma_{C}}
$$

where $\sigma_{M}\left(\sigma_{C}\right)$ denotes the standard deviation of mothers' (children's) employment. Because the variability of mothers' and children's employment is very similar, there is not a big difference between the reported regression coefficients and the correlation coefficients ${ }^{10}$ We hence present only the regression coefficients throughout the main text and refer to the coefficient of interest, $\beta$, as the correlation of intergenerational employment status. More details about methodological issues in measuring the intergenerational persistence of labor market outcomes can be found in Appendix B.2.

\section{Results}

\subsection{Intergenerational correlation of employment}

In this section, we document the intergenerational correlation of employment status for the United States. Table 2 shows the regression coefficients for maternal employment and covariates estimated using equation (1). Standard errors are clustered at the mother level to account for possible auto-correlation in siblings' error terms.

\footnotetext{
${ }^{9}$ Note that the correlation coefficient is conditional on covariates $X_{M i}$ and $X_{C i}$ if included in the regression.

${ }^{10}$ The standard deviations of the permanent components $l_{M i}$ and $l_{C i}$ are $\sigma_{M}=0.30$ and $\sigma_{C}=0.32$.
} 
The first column (without controls) shows an unconditional correlation of employment of 0.21. Children are on average employed for an additional 11 weeks when their mother is employed one more year $(0.21 \times 52 \approx 11) 11$ This finding of a substantial association in employment across generations is, to the best of our knowledge, a novel fact.

Table 2: Baseline regression

Dependent variable: Employment - child $\left(l_{C i}\right)$

\begin{tabular}{lcccc}
\hline Specification & $(1)$ & $(2)$ & $(3)$ & $\begin{array}{c}(4) \\
\text { Baseline }\end{array}$ \\
\hline Employment - mother $l_{M i}$ & $0.21^{* * *}$ & $0.14^{* * *}$ & $0.12^{* * *}$ & $0.12^{* * *}$ \\
& $(0.029)$ & $(0.028)$ & $(0.027)$ & $(0.027)$ \\
Ability - mother & & $0.05^{*}$ & 0.04 & 0.01 \\
& & $(0.029)$ & $(0.027)$ & $(0.027)$ \\
Ability - child & & $0.12^{* * *}$ & $0.07^{* * *}$ & $0.07^{* *}$ \\
& & $(0.029)$ & $(0.026)$ & $(0.026)$ \\
Yrs. schooling - mother & & 0.00 & 0.00 & -0.00 \\
& & $(0.004)$ & $(0.004)$ & $(0.004)$ \\
Yrs. schooling - child & & $0.02^{* * *}$ & $0.01^{* * *}$ & $0.01^{* * *}$ \\
& & $(0.003)$ & $(0.003)$ & $(0.003)$ \\
Net worth - mother & & & 0.01 & 0.00 \\
& & & $(0.005)$ & $(0.005)$ \\
Net worth - child & & & -0.01 & $-0.01^{*}$ \\
& & & $(0.006)$ & $(0.006)$ \\
Number of children - mother & & & & 0.00 \\
Adjusted $R^{2}$ & & & & $(0.006)$ \\
Number of children - child & & & & $-0.04^{* * *}$ \\
& & & & \\
Control age at birth - mother & NO & NO & NO & YES \\
\hline
\end{tabular}

Notes: Standard errors clustered at the mother level in parentheses. ${ }^{* * *} \mathrm{p}<0.01,{ }^{* *} \mathrm{p}<0.05,{ }^{*} \mathrm{p}<0.1$

In the remaining specifications, we further include covariates that typically influence the outcome variable, i.e. employment. In specification (2) we control for ability and education,

\footnotetext{
${ }^{11}$ As a comparison, estimates for the intergenerational elasticity of income for the US have oscillated around 0.4 in early work based on survey data (Solon, 1992, Zimmerman, 1992) to above 0.5 in recent work using administrative data (Chetty et al. 2014). Smaller figures correspond to other outcomes related to employment; for example, Toledo(2010) estimates 0.21 intergenerational correlation in hours, and Macmillan (2011), 0.1 for non-employment.
} 
of both mother and child. We observe that the main predictors, significant at the $1 \%$ level, are ability and education of the child. The mother's ability is significant at the $10 \%$ level, while her education is insignificant. Importantly, the coefficient on the mother's employment declines by one-third compared to specification (1), being 0.14 , still statistically significant at the $1 \%$ level.

In specification (3), we include net worth to control for potential wealth effects on labor supply. Although we observe that the sign on children's net worth is negative as expected, both coefficients are small and insignificant. The coefficient on employment of the mother declines only slightly, to 0.12 .

Finally, in specification (4) we additionally control for the number of children of both generations and the age of the mother at birth using dummies. This is the specification we will use in everything that follows, unless stated otherwise. The number of children is intended to control for correlated fertility attitudes, which in turn would affect labor supply. An additional grandchild reduces lifetime employment of the child by $4 \%$, statistically significant at the $1 \%$ level.

In all specifications from (2) to (4), the coefficient on the maternal employment is significantly positive. The value in the baseline specification with all the controls is 0.12 . Hence, an increase of the mother's employment by one year increases employment of the child by around six weeks $(0.12 \times 52 \approx 6)$. Human capital variables (education and ability) seem to play an important role in the intergenerational correlation of employment, as most of the difference between the coefficient of 0.12 in the regression with all the controls and the coefficient of 0.21 in the regression without controls occurs when these variables are included. However, there is a big part of the intergenerational correlation of employment that cannot be explained by either human capital or the other controls.

Extensive versus intensive margin of labor supply. In the baseline results in Table 2, we focus on the extensive margin of labor supply, the main interest of our investigation. To put these results into perspective, we include now a measure of intensive margin of labor supply: weekly working hours. Table 3 repeats the estimates of $\beta$ for employment status in the first two columns (specification (1) and (4) in Table 2). In the last two columns, we show the analogous coefficients of a regression using hours worked per week instead of employment (we include the periods of non-employment with zero hours worked).

The unconditional regression coefficients in columns one and three are both significantly positive. However, once we introduce the relevant controls in columns two and four, the 
Table 3: Margins of labor supply

Dependent variables: Employment - child and weekly hours - child

\begin{tabular}{|c|c|c|c|c|}
\hline \multirow[b]{2}{*}{ Employment - mother } & \multicolumn{2}{|c|}{ Employment - child } & \multicolumn{2}{|c|}{ Weekly hours - child } \\
\hline & $\begin{array}{c}0.21^{* * *} \\
(0.029)\end{array}$ & $\begin{array}{c}0.12^{* * *} \\
(0.027)\end{array}$ & & \\
\hline Weekly hours - mother & & & $\begin{array}{c}0.13^{* * *} \\
(0.031)\end{array}$ & $\begin{array}{c}0.04 \\
(0.031)\end{array}$ \\
\hline Controls & $\mathrm{NO}$ & YES & $\mathrm{NO}$ & YES \\
\hline Observations & 2,339 & 1,969 & 2,433 & 2,034 \\
\hline Adjusted $R^{2}$ & 0.04 & 0.09 & 0.01 & 0.06 \\
\hline
\end{tabular}

Notes: Standard errors clustered at the mother level in parentheses. ${ }^{* * *} \mathrm{p}<0.01,{ }^{* *} \mathrm{p}<0.05,{ }^{*} \mathrm{p}<0.1$. In columns two and four, we use the same covariates as in the baseline specification (4) in Table 2 ability, years of schooling, net worth, and number of children for mothers and children, as well as mother's age at birth.

coefficient in weekly hours is not significantly different from zero anymore, while it remains significantly positive for employment status. Therefore, while ability, education, wealth, and other observables completely explain the correlation in hours worked, a substantial part of the correlation in employment status remains unexplained.

Spousal employment. So far, we have focused exclusively on maternal labor supply variables. It is important to determine whether a father's labor supply choices also influence the employment status of the children. It may be that the unexplained association between employment of mothers and children is due to the influence of the father. Unfortunately, the NLSY79 is not designed to match fathers to their children. However, the data provide information on the employment status of spouses as reported by mothers, which we use as a proxy for father's employment.

The first column of Table 4 repeats the baseline result for the sub-sample in which we also observe the spousal employment status (specification (4) in Table 2). Column two shows the regression output, where, instead of the maternal employment, we regress child employment on the spouse's employment status and covariates. We observe no significant effect on the children's lifetime employment. In the third column, we include both the maternal and spousal employment status and observe that the coefficient on maternal lifetime employment is not different from the one in the baseline specification (4) in column one, whereas the coefficient on spousal employment is insignificant. Finally, when we also introduce an 
Table 4: Spousal employment status

Dependent variable: Employment - child $\left(l_{C i}\right)$

\begin{tabular}{lcccc}
\hline Employment - mother & $\begin{array}{c}0.13^{* * *} \\
(0.032)\end{array}$ & & $\begin{array}{c}0.13^{* * *} \\
(0.032)\end{array}$ & $\begin{array}{c}0.13^{* * *} \\
(0.032)\end{array}$ \\
Employment - spouse & & 0.05 & 0.04 & 0.06 \\
& & $(0.074)$ & $(0.077)$ & $(0.089)$ \\
Emp. - mother x Emp. - spouse & & & & 0.22 \\
& & & & $(0.215)$ \\
Controls & YES & YES & YES & YES \\
\hline Observations & 2,086 & 2,086 & 2,086 & 2,086 \\
Adjusted $R^{2}$ & 0.09 & 0.08 & 0.09 & 0.09 \\
\hline
\end{tabular}

Notes: Standard errors clustered at the mother level in parentheses. ${ }^{* * *} \mathrm{p}<0.01, * * \mathrm{p}<0.05,{ }^{*} \mathrm{p}<0.1$. In all columns, we use the same covariates as in the baseline specification (4) in Table 2; ability, years of schooling, net worth, and number of children for mothers and children, as well as mother's age at birth. The regressions correspond to the triplets spouse-mother-child for which a spouse is reported. Note that not all mothers report having a spouse in all the waves, nor are their spouses the same across waves.

interaction term between mothers' and spouses' employment status (fourth column), this coefficient is positive and large but not statistically significant because of considerably large standard errors. Nonetheless, it suggests that when the employment status of the mother and her spouse are similar, this has a positive effect on the child's employment.

Robustness. The main result of a positive and significant correlation between maternal and children's lifetime employment is robust to several changes in the specification. Variants in the specification are presented in more detail in the Appendix (Section B.3 explains additional details of some exercises, and the tables with results are shown in Section C.1). First, as is usual for the estimation of earnings correlations, we estimate equation (1) with logs of the permanent components (Table C.9). Second, following Chetty et al. (2014), we estimate rank-rank regressions for average employment status of mothers and children (Table C.10). Third, we adopt two alternatives in computing the permanent components: (i) simple averages of the employment status as the permanent component (without controlling for life-cycle or business-cycle fluctuations) as in the early literature (for example, Solon, 1992); and (ii) including controls for demographic events into the calculation of the permanent components (Table C.11). Finally, we also show that our results are robust to the use of other questions in the survey that allow for the inference of employment status but are less 
comparable across cohorts or less complete across years (Table C.12) 12

\subsection{Heterogeneous employment correlations}

In this section, we analyze whether the established fact of a significant and positive intergenerational correlation of lifetime employment differs across relevant dimensions, such as gender (daughters in comparison to sons) and socio-economic background (maternal education and income). We hence partition the sample in three different ways:

(i) according to the child's gender: $\mathcal{G}_{1}=\{$ sons, daughters $\}$

(ii) according to the highest formal maternal education: $\mathcal{G}_{2}=\{$ incomplete high school, complete high school, incomplete college, complete college\}

(iii) according to the mother's family income quintile: $\mathcal{G}_{3}=\{$ quintile $1, \ldots$, quintile 5$\}$

For all $k \in\{1,2,3\}$ the estimated models follow the specification,

$$
l_{C i}=\alpha+\sum_{G \in \tilde{\mathcal{G}}_{k}} \zeta_{G} \mathbb{I}_{i \in G}+\beta l_{M i}+\sum_{G \in \tilde{\mathcal{G}}_{k}} \beta_{G} \mathbb{I}_{i \in G} l_{M i}+\phi_{M} X_{M i}+\phi_{C} X_{C i}+\epsilon_{i}
$$

where the first group of each partition is our reference group (for example, sons in partition $\mathcal{G}_{1}$ ) and $\tilde{\mathcal{G}}_{k}$ denotes the partition without this first group (for example, $\tilde{\mathcal{G}}_{1}=\{$ daughters $\}$ ). The indicator variable $\mathbb{I}_{i \in G}$ takes the value one when child $i$ belongs to group $G$ and zero otherwise. In the following we discuss the coefficient $\beta_{G}$ and/or the marginal effect $\beta+\beta_{G}$ of mother's employment on the employment of children in the corresponding group $G$.

Gender. The first column of Table 5 shows the results of estimating equation (4) with $\mathcal{G}_{1}=\{$ daughters, sons $\}$. The coefficient on the interaction between employment of mothers and the daughter dummy is positive and statistically significant. The intergenerational correlation of employment is 0.18 for girls and 0.07 for boys ${ }^{13}$ Put differently, an increase in mothers' lifetime employment by one year increases employment of their daughters' by more than nine weeks on average, but their sons' employment by less than four weeks. The stronger link between mothers and daughters in terms of employment is interesting in light

\footnotetext{
${ }^{12}$ Further robustness exercises, such as using education-level dummies or including interactions of covariates, also confirm the findings of the baseline estimation. They are not included in the paper but are available upon request.

${ }^{13}$ Note that the coefficient for boys coincides with the marginal effect, as boys are the reference group in the regression. The numbers are the regression coefficients. The corresponding correlation coefficients (see equation (3) ) are 0.21 and 0.06 , respectively. The difference across genders increases as a consequence of disparities in standard deviations of lifetime employment.
} 
of the findings in the literature on intergenerational correlations of earnings that report lower estimates for daughters than for sons (see, for example, Chadwick and Solon, 2002; Olivetti and Paserman, 2015). It is also suggestive of a role-model effect, as role models are intuitively more likely to be gender specific. Nevertheless, the correlation between mothers' and sons' employment is still significantly positive, suggesting that the role-model effect exceeds a pure transmission of gender roles.

Table 5: Gender differences

Dependent variable: Employment - child $\left(l_{C i}\right)$

\begin{tabular}{lcc}
\hline & Equation (4) & Marginal effect \\
& & \\
\hline Employment - mother & $0.07^{* *}$ & $0.07^{* *}$ \\
& $(0.033)$ & $(0.033)$ \\
Employment - mother $\times$ Daughter & $0.11^{* *}$ & $0.18^{* * *}$ \\
& $(0.051)$ & $(0.041)$ \\
Controls & YES & \\
\hline Observations & 1,969 & \\
Adjusted $R^{2}$ & 0.11 & \\
\hline
\end{tabular}

Notes: Standard errors clustered at the mother level in parentheses; standard errors calculated using the delta method for the marginal effects. ${ }^{* * *} \mathrm{p}<0.01,{ }^{* *} \mathrm{p}<0.05,{ }^{*} \mathrm{p}<0.1$. In all columns, we use the same covariates as in the baseline specification (4) in Table 2; ability, years of schooling, net worth, and number of children for mothers and children, as well as mother's age at birth.

Maternal education. The intergenerational correlation of employment status is stronger the more disadvantaged the educational background of the mother. Figure 1 depicts the marginal effects of mothers' employment for each education level in $\mathcal{G}_{2}$. It is the highest and significantly positive for mothers with no degree (0.21) or a high-school degree (0.13). It is close to zero for mothers who attended college but did not complete it. Interestingly, if they obtained a college degree, the coefficient is again positive, suggesting a non-linearity in the transmission of employment status 14

Maternal family income. Since education is a crucial determinant of income, it should not be surprising that similar conclusions hold true when we consider maternal family income. Figure 2 shows the marginal effects of mothers' employment on children for each income quintile. The estimated coefficient is highest for children from mothers in the lowest income

\footnotetext{
${ }^{14}$ The corresponding regression results are reported in Table C.13 in the Appendix. It can be seen that the interaction of mothers' employment with incomplete college is statistically significant.
} 
Figure 1: Intergenerational correlation of employment status by maternal education

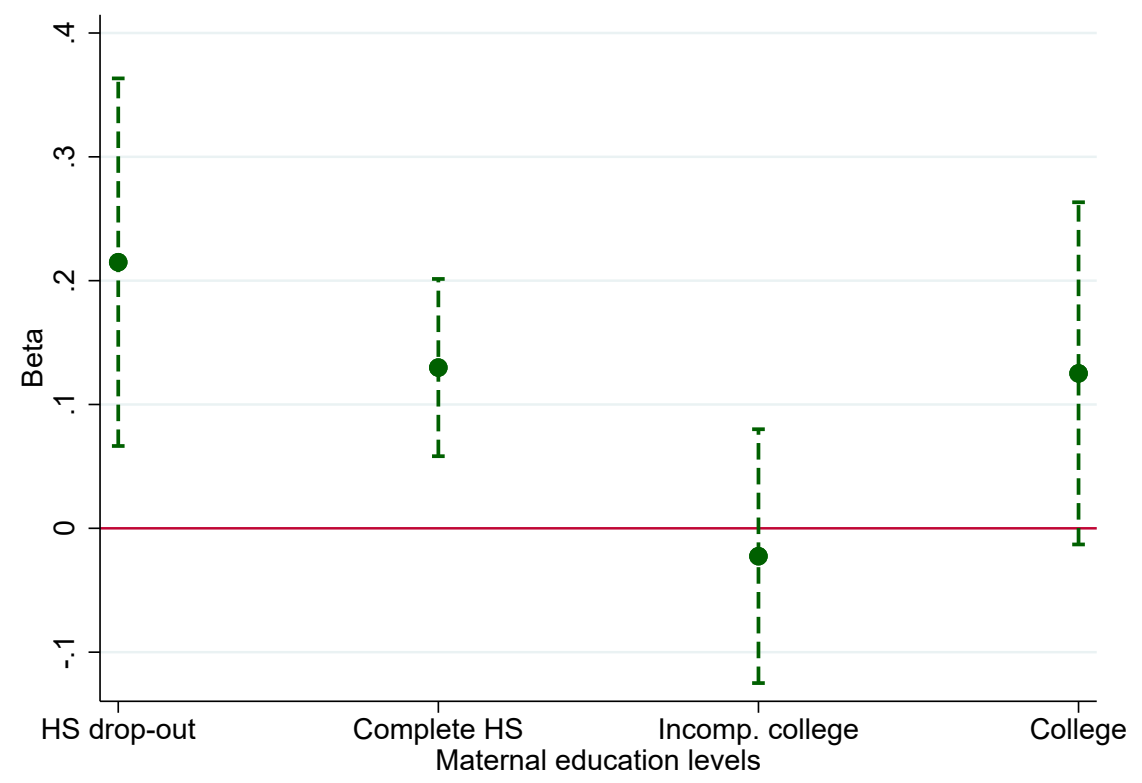

Notes: Standard errors clustered at mother level, determined using the delta method. 95\% confidence level intervals. The dependent variable is the permanent component of the employment status of the children. The maternal education is the maximum attained and observed education level. We use the same covariates as in the baseline specification (4) in Table 2 ability, years of schooling, net worth, and number of children for mothers and children, as well as mother's age at birth.

quintile (0.18). It then monotonically decreases in income, reaching zero at the fourth quintile. Interestingly, the point estimate for the fifth quintile is again positive. However, only for the first income quintile is the coefficient significant at the $5 \%$ level.

This pattern - a higher transmission of employment status at the bottom of the income distribution - is the same for daughters and sons, as Figure C.5 in the Appendix shows ${ }^{15}$ In particular, mothers from low-income families tend to transmit their employment status to their daughters much more than mothers with higher family income. By contrast, Olivetti et al. (2018) find that gender roles are transmitted more at the top of the income distribution. This discrepancy supports our claim that the residual employment correlation we document is not entirely the result of a transmission of gender roles.

The fact that the transmission of employment status is strongest for low-income earners is particularly interesting in light of existing income tax credits for low-income families with children, such as the EITC in the United States. Such programs directly encourage labor force participation of eligible recipients. If participation of these recipients is transmitted to their children (and hence their children's children, etc.), it may indirectly generate higher

\footnotetext{
${ }^{15}$ Figure C.5 further shows that education also affects the transmission of employment to girls and boys similarly.
} 
Figure 2: Intergenerational correlation of employment status by family income quintiles

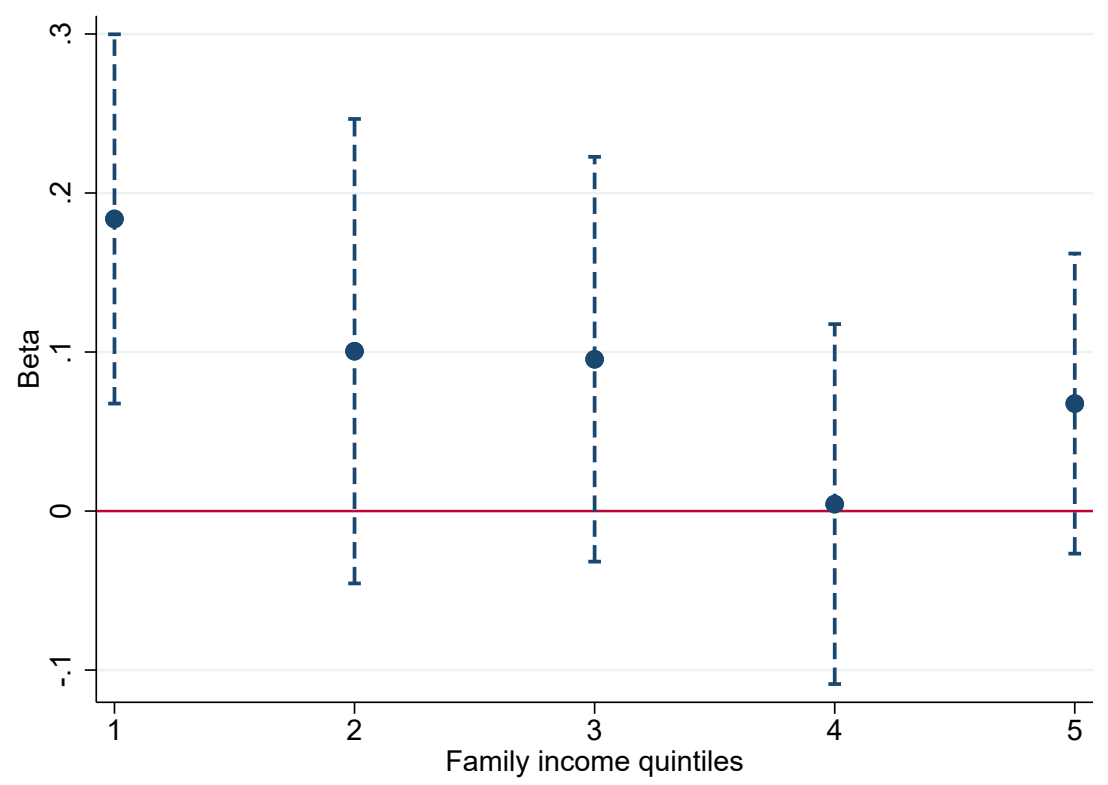

Notes: Standard errors clustered at mother level, determined using the delta method. $95 \%$ confidence level intervals. The dependent variable is the permanent component of the employment status of the children. Quintiles of family income correspond to the quintile observed in the majority of the survey years. We use the same covariates as in the baseline specification (4) in Table 2 ability, years of schooling, net worth, and number of children for mothers and children, as well as mother's age at birth.

labor income tax revenues in the following generations. Hence, there may be a dynamic fiscal benefit of such programs. However, before drawing normative conclusions from our - so far positive - analysis, it is necessary to get a better understanding of the precise mechanism through which employment status is transmitted. This is the focus of the remainder of this paper.

\section{$5 \quad$ Potential mechanisms}

In this section we evaluate potential mechanisms that could explain the significantly positive intergenerational correlation of employment status between mothers and their children. In the first part we discuss how far the transmission of attitudes toward work - or "work culture" - could explain the observed results. Particularly, we provide some evidence suggesting that there may be a role-model effect. Children who observe their parents working tend to have a more positive attitude towards work.

In the second part, we rule out several other mechanisms that could in theory explain the facts. Neither networks, occupation-specific human capital nor local labor markets seem 
to be a driving force behind the main result in Section 4 .

\section{$5.1 \quad$ Work culture}

One way to interpret the results is that employment of parents affects the attitude that children have towards work. In Appendix A we formalize this idea within a simple twogenerations model based on Solon (1999). In this model, we allow children's preferences to be affected by parental employment. The child's optimality condition is an intergenerational equation comparable to the one estimated above, with the coefficient on parent's (lifetime) employment precisely capturing this effect. A theory of work culture is hence consistent with the observed significant correlation between mothers' and children's employment.

As mentioned earlier, to answer normative questions it is necessary to understand the mechanism at work. Figure 3 is a schematic representation of two potential channels through which labor force participation of parents and that of the children are related. First, it could be that preferences are transmitted directly or "genetically". A mother who dislikes working tends to have children who dislike working. Second, it could be a role-model effect. Observing the mother participating in the labor market influences the child to develop a more positive attitude towards work.

This differentiation is important for policy analysis or dynamic scoring. For example, when evaluating the desirability of in-work benefits, only in the presence of a role-model channel will such benefits lead to higher income tax revenue raised from future generations. By contrast, if preference transmission does not operate through a role model, for example if children learn from what parents express or if genes play a role, such policies may increase the employment of mothers, but this increase will not spill over to their children and hence will have no effect on future income tax revenue.

Figure 3 illustrates these ideas. We observe a link between children's and parents' employment choices (purple line), and we infer that, after controlling for relevant observed factors (mainly ability, education, and wealth), there is a relation with preferences for work generating this link (red lines). The relation may arise either through direct preference transmission (relating parents' preferences and children's preferences directly) or through a role model (parents' employment choices influence children's preferences) or through a combination of both.

Disentangling the two potential channels is a difficult task because preferences are not directly observable. However, two questions in the NLSY79 are related to work preferences 
Figure 3: Direct preference channel versus role-model channel

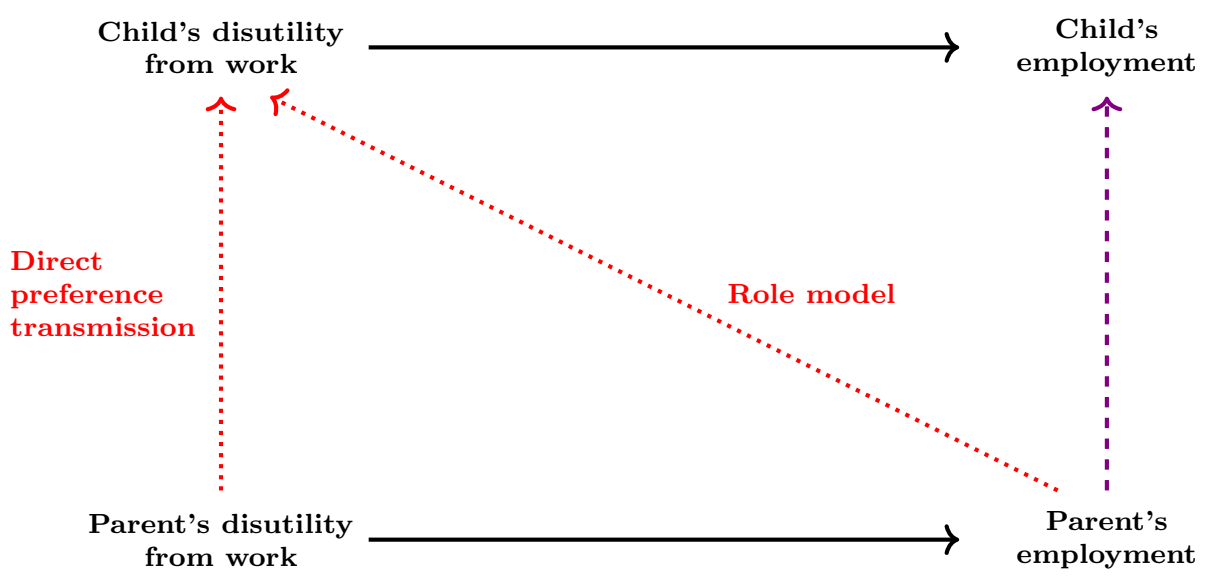

and we will make use of them in the following analysis: ${ }^{16}$

(i) Women's place is in the home, not in the office or shop.

(ii) Women are much happier if they stay at home and take care of the children.

While these survey questions relate foremost to gender roles, they also contain information on mothers' preferences for work. The answers in the survey are given qualitatively. We hence construct a quantitative variable, for which we code the answers of each question such that a higher value represents a higher disutility from work ${ }^{17}$

Figure 4 shows that there is indeed a sensible relationship between our constructed measure of disutility from work and the employment behavior of the mothers' cohort. The three lines correspond to the three terciles of our constructed measure. We observe the expected relationship: the distribution of the permanent employment component of mothers with low disutility from work has more mass at the right and less mass at the left than the distribution of the middle tercile; by contrast, the distribution conditional on high disutility of work has more mass at the left and less at the right.

Using our constructed measure for disutility of work $\theta_{M i}$, we then run the regression,

$$
l_{C i}=\alpha+\beta l_{M i}+\omega \theta_{M i}+\phi_{M} X_{M i}+\phi_{C} X_{C i}+\epsilon_{i} .
$$

Table 6 shows the results. The first column repeats the baseline estimation for comparison. The second column introduces our measure of disutility from work of the mother and excludes

\footnotetext{
${ }^{16}$ See Appendix B.4 for details. We use information only on the mothers because the analogous data for the children's cohort do not seem accurate, besides not being informative for male respondents.

${ }^{17}$ The resulting variable is directly comparable to the disutility parameter $\theta$ in the model of Appendix A
} 
Figure 4: Distribution of measure of maternal lifetime employment by levels of disutility from work

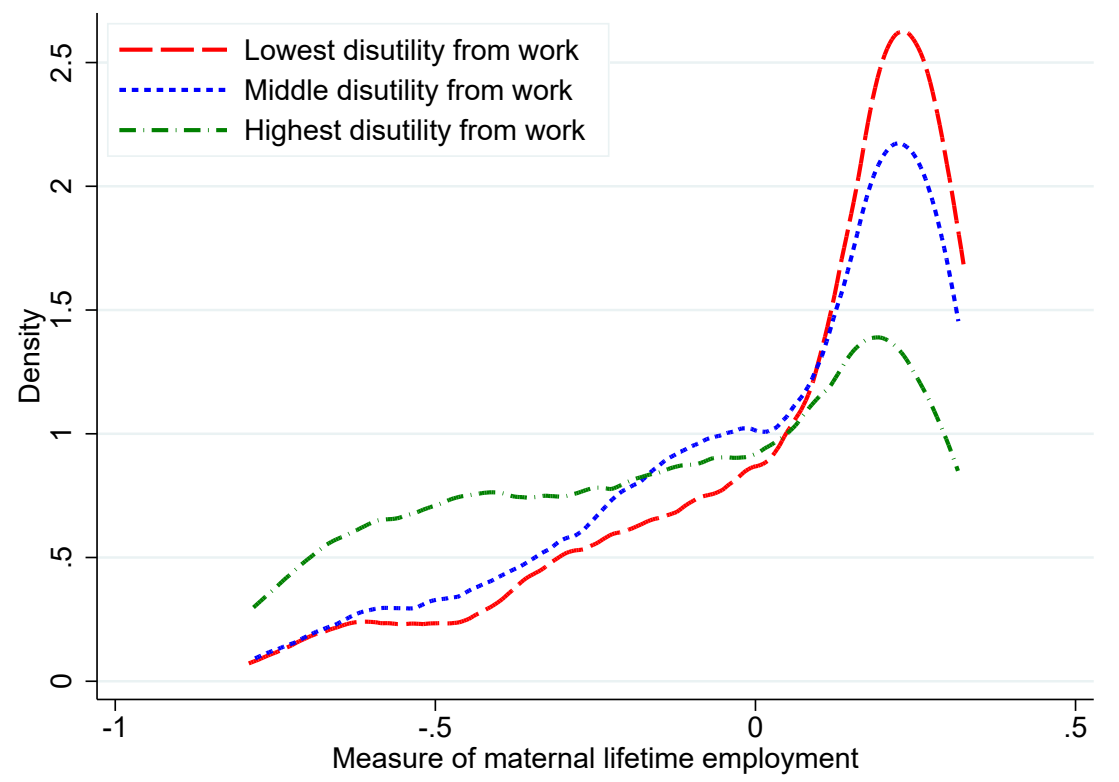

Notes: Disutility from work computed from questions on women's roles: (i) Women's place is in the home, not in the office or shop, and (ii) Women are much happier if they stay at home and take care of the children. Included in survey years 1979, 1982, 1987, and 2004. We assign the values: (a) strongly agree 1.5, (b) agree 0.5 , (c) disagree -0.5 , and (d) strongly disagree -1.5 . We average across questions and across years.

employment of the mother. The third column shows the results of including the preferences for work of the mother in our baseline specification, i.e. the estimation results of equation (5). The coefficient on employment of the mother does not change, and the coefficient on the disutility from work is close to zero. Finally, column four shows the same estimation but restricting the sample to daughters. The results are qualitatively the same, and the previous finding of a higher coefficient of maternal employment for daughter's employment behavior is confirmed. Again, the coefficient on mother's work preferences is close to zero and insignificant.

Importantly, while our measure of disutility from work is significantly negatively correlated with the employment behavior of mothers (the correlation coefficient is -0.27 , statistically significant at the $99 \%$ confidence level), it does not affect the employment behavior of children. Furthermore, including this measure in the baseline specification does not affect the coefficient on the mother's employment. These results suggest that the role-model channel is an important driver of the intergenerational correlation of employment, while there seems little or no direct transmission of work preferences.

Section B.5 in the Appendix shows one more indirect piece of evidence supporting the 
Table 6: Direct preference transmission vs. role model

Dependent variable: Employment - child $\left(l_{C i}\right)$

\begin{tabular}{lcccc}
\hline Specification & Baseline & Maternal preferences & Full & Only daughters \\
\hline Employment - mother & $0.12^{* * *}$ & & $0.12^{* * *}$ & $0.17^{* * *}$ \\
& $(0.027)$ & & $(0.027)$ & $(0.043)$ \\
Disutility from work - mother & & 0.02 & $0.03^{*}$ & 0.01 \\
& & $(0.014)$ & $(0.014)$ & $(0.022)$ \\
Controls & YES & YES & YES & YES \\
\hline Observations & 1,969 & 1,969 & 1,969 & 984 \\
Adjusted $R^{2}$ & 0.09 & 0.08 & 0.09 & 0.14 \\
\hline
\end{tabular}

Notes: Standard errors clustered at the mother level in parentheses. ${ }^{* * *} \mathrm{p}<0.01,{ }^{* *} \mathrm{p}<0.05,{ }^{*} \mathrm{p}<0.1$. We use the same covariates as in the baseline specification (4) in Table 2; ability, years of schooling, net worth, and number of children for mothers and children, as well as mother's age at birth. Disutility from work computed from questions on women's roles: (i) Women's place is in the home, not in the office or shop, and (ii) Women are much happier if they stay at home and take care of the children. Included in survey years 1979, 1982, 1987, and 2004. We assign the values: (a) strongly agree 1.5, (b) agree 0.5, (c) disagree -0.5, and $(\mathrm{d})$ strongly disagree -1.5 . We average across questions and across years.

importance of the role-model channel. Even when controlling for another proxy of maternal preferences for work, namely employment in periods the mother does not cohabit with the child, the role of the mother's employment remains relevant and predominant. The idea is that role models are transmitted when there is contact between mothers and children. Therefore, employment behavior in periods when children do not live with their mothers do not affect children's preferences for work via a role-model channel, but may convey information about maternal work preferences that are not perfectly correlated with their employment during cohabitation. Results show that employment when the mother and child do not cohabit does not have predictive power for the child's employment (see Table C.16).

\subsection{Mechanisms that do not work}

While the presented evidence suggests that work culture, or, more specifically, a role-model channel, is responsible for the observed intergenerational correlation in employment status, there are other factors that may well explain this correlation. In this section we briefly discuss three other candidate mechanisms and provide evidence that neither of them is likely to be the driving force behind the results. 


\subsubsection{Networks or occupation-specific human capital}

Parents might help children find a job through their connections, or even transmit occupationspecific human capital or preferences leading to correlations in job-finding probabilities across generations ${ }^{18}$ In order to test whether those mechanisms are plausible explanations for the residual intergenerational correlation of employment, we do the following. We split the sample between mother-child pairs who are employed in the same type of business (proxied by industry and sector) or have the same occupation, and those who have different industry/occupations 19 Industries, sector and occupation are assigned to the individuals according to the category observed most of the survey years. In particular, we estimate equation (4) using the partitions $\mathcal{G}_{4}=\{$ different industry-sector, same industry-sector $\}$ and $\mathcal{G}_{5}=\{$ different industry-occupation, same industry-occupation $\}$.

The first two columns of Table 7 show the results. They suggest that the correlation of employment is not different for mother-child pairs who share the same type of business or occupation. The estimation is imprecise because there are few observations for which child and mother share these traits (18\% for industry and sector, and $5 \%$ for industry and occupation). However, the point estimates suggest a pattern opposite to what a network channel or transmission of specific human capital would suggest: the correlation of employment is smaller when child and mother are in the same type of business or occupation. This evidence does not support a story of employment correlations driven by networks or specific human capital transmission.

\subsubsection{Local labor markets}

As a last exercise, we evaluate whether local conditions of the labor market could explain our correlation. So far, our argumentation has revolved around labor supply decisions. However, the estimated correlation could also be driven by market conditions that are determined by labor demand: if mothers and children live in the same region, both generations face similar labor market conditions, i.e. similar separation and job-finding probabilities.

The general version of the NLSY79 contains three different geographic variables but not a precise regional identifier. We hence undertake the following strategy. First, we condition

\footnotetext{
${ }^{18}$ The role of nepotism and preferences for occupations in the intergenerational correlation of earnings has been documented in the literature. See, for example, Corak and Piraino (2011) and Lo Bello and Morchio (2018).

${ }^{19}$ Industries according to the three-digit Census classifications are grouped in 14 aggregate categories, and a similar aggregation is done for occupations to 18 categories. The sectors considered are private, public, self-employment, and family businesses.
} 
our analysis on the mother-child pair living in the same broadly defined region 20 Second, we define a variable that indicates if both the mother and the child live in the same region as well as in an urban or rural area and in a Standard Metropolitan Statistical Area (SMSA).21 We assign residence according to the category observed in the majority of survey years, and we compute the intergenerational correlation of employment distinguishing mother-child pairs for which their categories coincide or not.

The last two columns of Table 7 present the estimates. Residence in the same location does not significantly affect the employment correlation. Again, the marginal effects for pairs that share geographical variables are smaller than the effects for pairs whose variables differ. Therefore, we do not find evidence that local labor markets can explain the significant intergenerational correlation of employment.

Table 7: Intergenerational correlation of employment status by (i) same industry-sector, (ii) same industry-occupation, (iii) same region, and (iv) same region-SMSA-urban/rural

Dependent variable: Employment - child $\left(l_{C i}\right)$

\begin{tabular}{lcccc}
\hline & $\begin{array}{c}\text { Industry- } \\
\text { sector }\end{array}$ & $\begin{array}{c}\text { Industry- } \\
\text { occupation }\end{array}$ & Region & $\begin{array}{c}\text { Region-SMSA- } \\
\text { urban/rural }\end{array}$ \\
\hline Employment - mother & $0.13^{* * *}$ & $0.12^{* * *}$ & $0.26^{* *}$ & $0.12^{* * *}$ \\
Employment - mother $\times$ Same & $(0.030)$ & $(0.028)$ & $(0.105)$ & $(0.030)$ \\
& -0.07 & -0.11 & -0.15 & -0.01 \\
Controls & $(0.059)$ & $(0.080)$ & $(0.109)$ & $(0.061)$ \\
& YES & YES & YES & YES \\
Observations & 1,969 & 1,969 & 1,969 & 1,969 \\
Adjusted $R^{2}$ & 0.09 & 0.09 & 0.09 & 0.09 \\
\hline
\end{tabular}

Notes: Standard errors clustered at the mother level in parentheses; standard errors calculated using the delta method for the marginal effects. ${ }^{* * *} \mathrm{p}<0.01,{ }^{* *} \mathrm{p}<0.05,{ }^{*} \mathrm{p}<0.1$. Industry, sector, occupation, region, SMSA and urban/rural are assigned as the category that is observed in the majority of the survey years. In all columns, we use the same covariates as in the baseline specification (4) in Table 2; ability, years of schooling, net worth, and number of children for mothers and children, as well as mother's age at birth.

\footnotetext{
${ }^{20}$ The variable region indicates whether the individual lives in one of four areas, Northeast, North Central, South or West. 93\% of the mother-child pairs share the region of residence.

${ }^{21}$ The measure is still imperfect because it could be that both live in an urban area within the same broad region and in an SMSA that could be a different metropolitan city. But only $24 \%$ of the observations correspond to pairs living in the same combination of geographical variables.
} 


\section{Conclusion}

This paper contributes to the literature on the intergenerational correlation of labor market outcomes. Differently from the existing literature, we focus on the extensive margin of labor supply. Using the NLSY79 and the CNLSY79 we document a robust, statistically significant, and positive intergenerational correlation of employment status between mothers and their children. After controlling for the channels that drive the transmission of earnings potential, we find that an increase in lifetime employment of mothers by one year is associated with an increase in the employment of her child by six weeks. The correlation is higher for mother-daughter pairs than for mother-son pairs. Furthermore, it is lower when the maternal education and the family income are higher.

While the analysis of this paper is a purely positive one, it has potentially important normative implications. For example, in-work benefits, such as the EITC in the United States, paid to the currently working generation may indirectly increase the employment - and thus income tax revenue - of future generations. This is especially the case if these programs are targeted to low-income families with children. More generally, dynamic scoring of any redistributive policy that affects incentives to work should take this transmission channel into account.

However, a comprehensive policy analysis requires a clear understanding of the mechanism, through which employment status is transmitted across generations. We show that the results are consistent with a theory of work culture and provide suggestive evidence that in their employment decisions, mothers act as a role model for their children, especially for their daughters. We are able to rule out network effects, occupation-specific human capital, and local labor markets as driving forces behind the result.

\section{References}

Abbott, B., G. Gallipoli, C. Meghir and G. Violante (2013): Education policy and intergenerational transfers in equilibrium, Technical report, NBER Working Paper 18782.

Altonji, J. and T. Dunn (2000): An intergenerational model of wages, hours, and earnings, Journal of Human Resources 35 (2), 221-258.

Binder, A. (2018): Inter-generational transmission and the 21st century rise in skilled mothers' labor supply, Technical report, University of Michigan. 
Björklund, A. and M. Jäntti (2011): Intergenerational income mobility and the role of family background, In: B. Nolan, W. Salverda and T. Smeeding (Eds.), Oxford Handbook of Economic Inequality, Elsevier, 1-36.

Black, S. and P. Devereux (2011): Recent developments in intergenerational mobility, In: O. Ashenfelter and D. Card (Eds.), Handbook of Labor Economics, Volume 4, Part B, Elsevier, 1487-1541.

Bowles, S. and H. Gintis (2002): The inheritance of inequality, Journal of Economic Perspectives 16 (3), 3-30.

Chadwick, L. and G. Solon (2002): Intergenerational income mobility among daughters, American Economic Review 92 (1), 335-344.

Chetty, R., Hendren N., P. Kline and E. Saez (2014): Where is the land of opportunity? The geography of intergenerational mobility in the United States, Quarterly Journal of Economics 129 (4), 1553-1623.

Corak, M. and P. Piraino (2011): The intergenerational transmission of employers, Journal of Labor Economics 29 (1), 37-68.

Dahl, G. and A. Gielen (2018): Intergenerational spillovers in disability insurance, Technical report, IZA Discussion Paper N. 11334.

Dahl, G., A. Kostøl and M. Mogstad (2014): Family welfare cultures, Quarterly Journal of Economics 129 (4), 1711-1752.

Dahl, G., K. Loken and M. Mogstad (2014): Peer effects in program participation, American Economic Review 104 (7), 2049-2074.

Fernandez, R. (2007): Women, work, and culture, Journal of the European Economic Association 5 (2-3), 305-332.

Fernandez, R. (2013): Cultural change as learning: The evolution of female labor force participation over a century, American Economic Review 103 (1), 472-500.

Fernandez, R. and A. Fogli (2009): Culture: An empirical investigation of beliefs, work, and fertility, American Economic Journal: Macroeconomics 1 (1), 146-177. 
Grawe, N. (2006): Lifecycle bias in estimates of intergenerational earnings persistence, Labour Economics 13 (5), 551-570.

Haider, S. and G. Solon (2006): Life-cycle variation in the association between current and lifetime earnings, American Economic Review 96 (4), 1308-1320.

Hartley, R., C. Lamarche and J. Ziliak (2017): Welfare reform and the intergenerational transmission of dependence, Technical report, IZA Discussion Paper N. 10942.

Lee, C. and G. Solon (2009): Trends in intergenerational income mobility, Review of Economics and Statistics 91 (4), 766-772.

Lo Bello, S. and I. Morchio (2018): Like father, like son: Occupational choice, intergenerational persistence and misallocation, Technical report, Mimeo.

Lochner, L., Y. Park and Y. Shin (2018): Wage dynamics and returns to unobserved skill, Technical report, NBER Working Papers 24220.

Macmillan, L. (2011): Measuring the intergenerational correlation of worklessness, Technical report, CMPO Working Paper N. 11/278.

Mazumder, B. (2005): Fortunate sons: New estimates of intergenerational mobility in the United States using social security earnings data, Review of Economic and Statistics 87 (2), 235-255.

Nybom, M. and J. Stuhler (2016): Heterogeneous income profiles and lifecycle bias in intergenerational mobility estimation, Journal of Human Resources 51 (1), 239-268.

Nybom, M. and J. Stuhler (2017): Biases in standard measures of intergenerational income dependence, Journal of Human Resources 52 (3), 800-825.

Olivetti, C. and D. Paserman (2015): In the name of the son (and the daughter): Intergenerational mobility in the United States, 1850-1940, American Economic Review 105 (8), 2695-2724.

Olivetti, C., E. Patacchini and Y. Zenou (2018): Mothers, peers and gender-role identity, Journal of the European Economics Association, forthcoming.

Solon, G. (1989): Biases in the estimation of intergenerational earnings correlations, Review of Economics and Statistics 71 (1), 172-174. 
Solon, G. (1992): Intergenerational income mobility in the United States, American Economic Review 82 (3), 393-408.

Solon, G. (1999): Intergenerational mobility in the labor market, In: O. Ashenfelter and D. Card (Eds.), Handbook of Labor Economics, Volume 3, Elsevier, 1761-1800.

Toledo, M. (2010): On the intergenerational persistence of work hours, Technical report, Mimeo.

Zimmerman, D. (1992): Regression towards mediocrity in economic stature, American Economic Review 82 (3), 409-429. 


\section{Appendix}

\section{A Two-generations model}

The model is a simple two-generations framework based on Solon (1999). The main addition to it is that children's preferences towards work are (potentially) affected by parental labor force participation.

There is a continuum of families, each consisting of one parent and one child 22 Generations are indexed by $k \in\{M, C\}$ for parents and children, respectively. Parents are altruistic but discount their child's expected utility by a factor $\alpha \in[0,1)$. They decide on consumption $c_{M}$, labor supply $l_{M}$, and human capital investment for their child $H$. Children decide on consumption $c_{C}$ and labor supply $l_{C}$, but they do not have any offspring and hence do not invest in human capital. Agents are heterogeneous in ability $e_{k}$ and disutility from labor $\theta_{k} 23$ Abilities are correlated across generations, accounting for genetic inheritance.

The parents' optimization problem is given by

$$
\begin{aligned}
V_{M}\left(\theta_{M}, e_{M}, v_{M}\right)= & \max _{c_{M}, l_{M}, H} \frac{c_{M}^{1-\sigma}}{1-\sigma}-\theta_{M} \frac{l_{M}^{1+\chi}}{1+\chi}+\alpha \mathbb{E}\left[V_{C}\left(\theta_{C}, w_{C}\right)\right] \\
\text { s.t. } \quad & c_{M}+p H=w_{M} l_{M} \\
& \log \left(w_{M}\right)=\log \left(e_{M}\right)+v_{M} \\
& \log \left(\theta_{C}\right)=\kappa_{0}-\kappa_{1} \log \left(l_{M}\right)+\eta_{C} .
\end{aligned}
$$

We assume that utility is additively separable in consumption and labor. The parameter $\sigma>0$ is the coefficient of relative risk aversion and $\chi>0$ is the inverse of the Frisch elasticity of labor supply. Parents finance consumption $c_{M}$ and investment in their child's human capital $H$, a unit of which costs $p$, with labor earnings $w_{M} l_{M}$. The wage of the parent is determined through ability $e_{M}$ and a random term $v_{M}$, which captures labor market luck.

The last equation (6) is the process of intergenerational transmission of preferences for work. Children's disutility from labor, $\theta_{C}$, (potentially) depends on the parental labor supply decision $l_{M}$, through a parameter $\kappa_{1}$. A value of $\kappa_{1}$ different from zero means that parents' labor supply has an effect on children's preferences for work. We do not impose any prior

\footnotetext{
${ }^{22}$ The exposition of the model uses the word parent for the sake of generality, even if we use mothers in the empirical analysis. For consistency with the notation in the empirical setup, we denote the parents with the indicator $M$.

${ }^{23}$ Whereas differences in productivity among children are captured explicitly by both $e_{C}$ (ability) and $H$ (education), $e_{M}$ represents for parents a combination of abilities and education, the latter not being modeled.
} 
on the direction of the effect. If $\kappa_{1}>0$, then the more parents work, the less children dislike working, and the opposite is the case for $\kappa_{1}<0$. If $\kappa_{1}=0$, then parental employment does not have any influence on children's preferences for work. The parameter $\eta_{C}$ is an idiosyncratic preference shock.

Similarly, the child's optimization problem is given by

$$
\begin{aligned}
V_{C}\left(\theta_{C}, w_{C}\right)= & \max _{c_{C}, l_{C}} \frac{c_{C}^{1-\sigma}}{1-\sigma}-\theta_{C} \frac{l_{C}^{1+\chi}}{1+\chi} \\
\text { s.t. } \quad & c_{C}=w_{C} l_{C} \\
& \log \left(w_{C}\right)=\log \left(e_{C}\right)+\psi \log (H)+v_{C} \\
& \log \left(e_{C}\right)=\lambda \log \left(e_{M}\right)+u_{C} .
\end{aligned}
$$

Children finance their consumption with labor earnings. Wages $w_{C}$ of children depend on their ability, $e_{C}$, on the acquired human capital $H$ (which has a return $\psi$ ), and $v_{C}$, which captures labor-market luck. The last equation states that ability is partially inherited. To be specific, the parent's and child's ability are linked via an AR(1) process with persistence $\lambda \in(0,1)$.

Note that in the model, $l_{M}$ and $l_{C}$ are continuous variables, although we focus on the extensive margin of labor supply. In the model, we think of $l_{M}$ and $l_{C}$ as the time share in employment over the whole lifetime. This maps well into our empirical analysis, in which we employ the permanent component of employment status.

The Solution. We focus on the solution of the children's problem because it enables us to summarize the relevant model predictions. To be specific, we take parental decisions and realizations of shocks as given. Then, the first-order condition for labor supply $l_{C}$ can be written as

$$
\log \left(l_{C}\right)=-\frac{1}{\sigma+\chi} \log \left(\theta_{C}\right)+\frac{1-\sigma}{\sigma+\chi} \log \left(w_{C}\right)
$$

We can substitute for $\log \left(\theta_{C}\right)$ with (6) and $\log \left(w_{C}\right)$ with (9) and obtain

$$
\log \left(l_{C}\right)=\alpha+\beta \log \left(l_{M}\right)+\gamma \log \left(e_{M}\right)+\delta \log (H)+\epsilon,
$$

where the coefficients $\alpha, \beta, \gamma$ and $\delta$ are functions of structural model parameters. 
Specifically,

$$
\beta=\frac{\kappa_{1}}{\sigma+\chi}
$$

This resulting intergenerational equation of employment status 12 is similar in many respects to the models we estimated in Section 4.1. It relates children's and parents' employment decisions once human capital decisions and ability transmission have been taken into account. Importantly, employment decisions conditional on human capital and ability are related across generations through the coefficient $\beta$. $\beta$ is proportional to, and has the same sign as, $\kappa_{1}$, which determines how parents' labor supply translates into children's attitude towards work. Equation 12 thus provides an empirical test for the presence of the transmission of preferences for work. Because in our estimation $\beta>0$, according to our theory the child's disutility from work decreases with parental labor supply.

Although the essence of the solution $(12)$ coincides with the type of estimated models in Section 4.1 (see Table 2), there are some differences. Apart from some factors not present in the model, for simplicity (for example, wealth, fertility), the specification in the model is in logs, whereas the empirical specification is linear. This choice responds to simplicity both in the model and in the empirical estimation 24 As we showed already, the empirical results are robust to a vast set of changes in the specification.

\section{B Details on the empirical analysis}

\section{B.1 Details on the data}

NLSY79 and CNLSY79. The data is collected and provided freely by the Bureau of Labor Statistics (BLS) in the US. The NLSY79 consists of three sub-samples: (i) the cross-sectional sample (6,111 individuals) is a representative sample of the US population in 1979, (ii) the supplemental sample (5,295 individuals) over-samples disadvantaged groups (Hispanic or Latino, black and poor people), and (iii) the military sample (1,280 individuals) over-samples the population participating in the army. As explained in the main text, we use only the cross-sectional sample and restrict ages to 25 to 45 years old. Figure C.1 provides an example

\footnotetext{
${ }^{24}$ Using the linear relationship has the advantage of avoiding arbitrary transformations of the data. Not all permanent components are above 0 . Hence, to be able to use the log-specification, we need to shift all permanent components to ensure that they are above 0. But these shifts complicate the interpretation of the coefficients because they are not invariant to the size of the shift. Furthermore, the interpretation of results is very intuitive in the linear setup.
} 
for a mother-child pair in the data.

It is worth noting some features of the sample we use for the analysis. Figure C.2 shows the distribution of the number of interviews. The mode for mothers is 15 , with around $75 \%$ of the mass concentrated between 14 and 16 interviews. For children, the mode is 3, and only $50 \%$ have 3 or more interviews. The left panel of Figure C.3 shows the distribution of the age of mothers at birth. Of the observations, $80 \%$ come from mothers who gave birth between 20 and 23 years old. The right panel of Figure C.3 shows the same distribution, broken down by number of interviews of the children. Mothers of children with more interviews were younger when their children were born. Figure C.4 shows the employment-age profiles of mothers and children. The composition of the children's sample, biased towards younger children, as explained in the main text, is also behind the atypical employment-age profile for the cohort. Employment rates decline and become more volatile with age because older children are fewer and belong to mothers who were younger at birth, something the empirical strategy accounts for when computing the permanent components. Furthermore, the dip in the employment rate at the age of 35 to 36 for children reflects the 2008 crisis, which particularly affected younger cohorts.

Ability is measured in the 1979 cohort by the Armed Services Vocational Aptitude Battery (ASVAB), which was collected around 1980 when mothers were between 15 and 23 years old. The scores correspond to the AFQT, which is a composite of test results in arithmetic reasoning, word knowledge, paragraph comprehension, and numerical operations. We use the version of the AFQT revised in 2006 to control for differences in cohorts within the NLSY79. Similar measures of cognitive abilities have been collected for the children cohort since 1986. In particular, we use the latest measurement for each child of the Peabody Individual Achievement Test (PIAT) for Math, considered the most appropriate measure of ability among the test scores available in the data for the younger cohort Abbott et al., 2013). These measures may capture not only genetic ability, but also some components of scholastic skills. This is not a problem for our analysis, as we are interested in accounting for productivity jointly with education.

Another relevant variable in the analysis, wealth, is introduced as net worth, i.e. assets minus debts. The variable is provided by the BLS for the NLSY79 cohort, and we follow the definition in the CNLSY79, where such a computed variable is not provided. In terms of assets, we include savings in liquid accounts and in financial assets, the market value of the main house and other properties, and the market value of own vehicles. The debts 
comprise credit card balances, outstanding mortgage value and other property debts, debts for vehicles, and other debts. The net worth variable constructed by the BLS uses imputed assets and debts when there is no response, and values are top-coded. No such procedures are followed in the children's cohort, and also there are some slight changes in the definitions of assets and debts over time.

Earnings is also a variable used throughout the analysis. We use an annual measure, the most comparable variable across cohorts: wages and salaries received during the last calendar year. Earnings are top-coded for both the parents' and children's cohorts. We construct weekly hours of work, dividing total annual hours by total number of weeks worked during the last calendar year for the mothers' cohort. For the children's cohort, we use weekly hours worked in all jobs, as reported in the survey.

Industries are available according to different versions of the three-digit US Census classification. For the comparison of industries across generations, they are grouped into 14 categories: agriculture, forestry, fisheries; mining; construction; manufacturing of nondurables; manufacturing of durables; transportation, communications, and other public utilities; wholesale trade; retail trade; finance, insurance, and real estate; business and repair services; personal services; entertainment and recreation services; professional and related services; public administration. Similarly, the classification of occupations also corresponds to three-digit US Census classification. They are collapsed into 18 categories: management, business, and financial operations; computer and mathematical; architecture and engineering; life, physical, and social services; community and social services; legal; education, training, and library; arts, design, entertainment, sports, and media; health-care practitioners and technical and support; protective service; food preparation and serving related; building and grounds cleaning and maintenance; personal care and service; sales and related; office and administrative support; farming, forestry, and fishing; construction and extraction, installation, repair and maintenance, and production; transportation and material moving. The variable accounting for sectors refers to private, public, self-employment, and family businesses.

The geographical information on the publicly available version of the NLSY79 is not very detailed. The variables are limited to region (Northeast, North Central, South, or West), urban or rural, and an indicator of residence in an SMSA, which are highly populated areas. Whenever we need to construct a measure of "location," we use a combination of these three variables. 


\section{B.2 Methodological challenges in the measurement of intergener- ational persistence of labor market outcomes}

The data we use feature desirable characteristics for coping with some estimation issues identified in the literature on the intergenerational correlation of earnings. First, Zimmerman (1992) and Solon (1992) show that early estimations based on single-year measures of parents' and children's outcomes are subject to substantial measurement error. This is because single-year measures are subject to transitory deviations from the long-run means. This means that single-year measures are not good proxies for lifetime or permanent components, which yields attenuation bias as a consequence. This problem is particularly relevant for parental outcomes, the explanatory variables in the intergenerational equations. Mazumder (2005) estimates the potential reduction in the bias by increasing the number of observations. The longitudinal nature of the NLSY79 allows for the use of several observations for both generations, particularly in the case of mothers, who are observed on average in 14 waves in our sample (only $4 \%$ of the sample has fewer than 10 interviews).

Second, the lack of heterogeneity in the samples aggravates the measurement error (Solon, 1992, 1999) 25 We use a representative sample of the US population in 1979, namely the cross-sectional sub-sample of the NLSY79, which is several times bigger than cohorts formed from the Survey Research Center (SRC) component, the analogous of the PSID typically employed in empirical studies of intergenerational earnings' correlations (see, for example, Solon, 1992).

Finally, the literature emphasizes a life-cycle bias that arises when parents' and children's observations are not representative of their lifetime outcomes due to non-stable trajectories along the life (Haider and Solon, 2006; Grawe, 2006; Nybom and Stuhler, 2016, 2017). Measurement error is not homogenous along the life cycle, with higher noise for early and late years (Mazumder, 2005). To mitigate this problem, the literature recommends using observations for ages between 30 and 50 (Black and Devereux, 2011). Our sample restriction to individuals between 25 and 45 years old and the netting out of age effects from the permanent components are intended to mitigate this bias.

\footnotetext{
${ }^{25}$ The interaction between, on the one hand, transitory fluctuations and measurement error, and, on the other hand, the homogeneity in the sample, is discussed in Solon (1989).
} 


\section{B.3 Details on the robustness exercises}

In order to provide scale-invariant estimates of the persistence in employment, we follow the literature by providing a log-log and a rank-rank specification. It is worth noting that for the log-log specification, we take the logarithm of the permanent components, which are the fixed effects backed out in the estimation of (2). As these permanent components include negative values, to take the natural logarithm we add a constant such that the minimum value for each generation is 0.001 . For the rank-rank specification, we sort individuals within each generation in ascending order in terms of proportion of periods employed during the 25 to 45 years old window. We assign each individual their position, divided by the total number of individuals (when an employment value is repeated, we average across positions corresponding to that value).

For the robustness exercise, in which we control for demographic events when computing the permanent components, we estimate the following slightly modified model,

$$
l_{k i t}=l_{k i}+\sum_{n=1}^{2} \pi_{n k} A_{k i t}^{n}+\lambda_{k t}+D_{e m o}^{\prime}{ }_{k i t} \varsigma+v_{k i t},
$$

where $k \in\{M, C\}$ and $D e m o_{k i t}$ are controls for demographic events, including births, couple formation and dissolution, job loss and finding by partner, presence of children 0 to 3 years old in the household with/without child care, and presence of older children in the household. We also include controls for education level, region, urban area, living in own dwelling, conjugal status, and whether the partner works.

The alternative variables used to measure employment status are (i) the preferred employment questions without including the requirement of a minimum time or earnings as in the main estimation; (ii) answers to the Current Population Survey (CPS)-type employment status question in the mothers' cohort, and response to whether they have any employer at the time of the survey, for the children's cohort; (iii) questions about hours and earnings (employment corresponds to a positive number of hours and earnings, in the last year for the mother's cohort, and in the year of the survey for the children); and (iv) questions about hours only (last year for mothers, current year for children). As discussed, these questions are less comparable across generations than our preferred measure, and are only available for fewer periods. We also include labor force status for mothers, for whom unemployment questions are also available (this is not the case for the children's cohort). 


\section{B.4 Details on the preferences for work in NLSY79 and CNLSY79}

As referred to in the main text, the questions about women's roles that provide information on preferences for work are (i) Women's place is in the home, not in the office or shop, and (ii) Women are much happier if they stay at home and take care of the children. The questions are included in survey years 1979, 1982, 1987, and 2004 for mothers and 1994, 1996, 1998, 2002, 2006, and 2010 for children. These are qualitative questions, which we quantify with a range centered at zero. We assign the following values: (a) strongly agree 1.5, (b) agree 0.5, (c) disagree -0.5, and (d) strongly disagree -1.5. We average across the two questions for each year and across the years 26

Figure C.6 depicts the distribution of the resulting variable of maternal disutility from work. It is slightly skewed to the right, which means that there is an over-representation of mothers with low disutility from work, which is in agreement with a considerably high employment rate $(73 \%)$.

Furthermore, we take terciles of the variable, which gives us three classes that we describe as low, medium, and high maternal preferences for work. Summary statistics for the disutility from work by terciles are shown in Table C.14.

Table C.15 shows that whereas the proportion of children employed clearly varies with the employment of the mother, it does not show the same gradient with respect to the maternal disutility from work.

\section{B.5 Additional evidence favoring role model: Employment during non-cohabitation}

We perform one additional exercise supporting the evidence that favors a role-model over a direct preference transmission channel in explaining the intergenerational correlation of employment. We attempt to proxy for the preferences for work of mothers to include in the regression specified in equation (5), using employment of the mother - the permanent component - only in the years when the child does not cohabit with her (before the child was born, or after the child left the household). The idea is that the role model can be transmitted only when children observe the behavior of their mothers, and this is facilitated during

\footnotetext{
${ }^{26}$ If information on a variable is missing in a year, we use only the available information for the other variables for that year. This way, we put equal weight on all years. We checked that alternatives (either averaging only the information on the first or the second question) do not change the results. Our preferred measure of disutility from work is negatively correlated with the permanent component of employment (the correlation is -0.27 unconditional, and -0.15 once we controlled for all the covariates in the previous regressions, both statistically significant at the $99 \%$ confidence level).
} 
cohabitation. Therefore, mothers' employment during periods of non-cohabitation would control for the work preferences of the mother herself when added to lifetime employment behavior.

But periods when children do or do not live with their mothers are in general at different points of the maternal life cycle. Even though we control by age for computing the permanent components, if there is not enough variation in mothers' ages during cohabitation and noncohabitation, we might suffer mis-measurement.

For each child, we split the observations of the child's mother into those when they are both cohabiting and those when they are not. Periods with or without cohabitation depend on the child. Non-cohabitation includes periods before the child's birth and after the child leaves the home, independent of whether other children are living in the household. We estimate the permanent component for mothers using only the latter period, and re-estimate the intergenerational equation introducing this variable to control for mothers' preferences for work. Note that we use only mother-child pairs that count with both observed periods of cohabitation and non-cohabitation.

Table C.16 essentially reproduces Table 6, but uses the permanent component of the mother's employment for periods when she does not cohabit with the child (specific to the mother-child pair) to control for the mother's preferences for work. The sign is the opposite of our variable of disutility from work, as employment is negatively associated to such disutility. The conclusions from this exercise are similar. The role of a mother's lifetime employment behavior continues to be relevant even after we control for her behavior when she is not cohabiting with the child. Furthermore, these periods of non-cohabitation do not seem to add information once lifetime employment is taken into account. This supports the preponderance of the role-model channel. 


\section{Additional tables and figures}

\section{C.1 Additional tables}

Table C.8: Additional summary statistics for women and mother-child pairs in NLSY79 and CNLSY79

\begin{tabular}{|c|c|c|c|}
\hline & Women & Mothers & Children \\
\hline White & $80 \%$ & $74 \%$ & $73 \%$ \\
\hline Black & $13 \%$ & $18 \%$ & $19 \%$ \\
\hline Migrant & $5 \%$ & $5 \%$ & $0 \%$ \\
\hline Public sector employees & $10 \%$ & $9 \%$ & $4 \%$ \\
\hline Private sector employees & $87 \%$ & $86 \%$ & $92 \%$ \\
\hline Self-employed & $3 \%$ & $4 \%$ & $2 \%$ \\
\hline Father at home & & & $56 \%$ \\
\hline Living in own dwelling & $92 \%$ & $94 \%$ & $74 \%$ \\
\hline Partner works & $64 \%$ & $68 \%$ & $51 \%$ \\
\hline Children 0 to 3 y.o. not in child care & $17 \%$ & $20 \%$ & $23 \%$ \\
\hline Children 0 to 3 y.o. in child care & $9 \%$ & $9 \%$ & $9 \%$ \\
\hline Children 4 to 5 y.o. & $17 \%$ & $23 \%$ & $19 \%$ \\
\hline Children 6 to 12 y.o. & $40 \%$ & $61 \%$ & $29 \%$ \\
\hline Children 13 to 15 y.o. & $15 \%$ & $26 \%$ & $3 \%$ \\
\hline Children 16 to 18 y.o. & $11 \%$ & $20 \%$ & $1 \%$ \\
\hline Births & $14 \%$ & $14 \%$ & $18 \%$ \\
\hline Couple dissolution & $4 \%$ & $5 \%$ & $7 \%$ \\
\hline Couple formation & $6 \%$ & $4 \%$ & $18 \%$ \\
\hline Partner job loss & $5 \%$ & $5 \%$ & $6 \%$ \\
\hline Partner job finding & $6 \%$ & $5 \%$ & $16 \%$ \\
\hline Individuals & 3,040 & 1,373 & 2,339 \\
\hline
\end{tabular}

Notes: Percentages for observations in the 25 to 45 years old range in our sample. For the sector of employment, the category most often observed is assigned to the individual. Similar criterium applies for the variable regarding the father living at home. The variables living in own dwelling, partner works, children of different ages, births, couple dissolution and formation, and partner job loss and job finding capture the number of observations for which they take the value 1 (the event occurs); they help understanding the nature of our sample. 
Table C.9: Robustness: Log-log regressions

Dependent variable: Log-employment - child $\left(\log \left(l_{C i}\right)\right)$

\begin{tabular}{lcccc}
\hline Specification & $(1)$ & $(2)$ & $(3)$ & $(4)$ \\
\hline Log-employment - mother & $0.21^{* * *}$ & $0.15^{* * *}$ & $0.15^{* * *}$ & $0.14^{* * *}$ \\
& $(0.034)$ & $(0.036)$ & $(0.037)$ & $(0.034)$ \\
Ability - mother & & 0.08 & 0.05 & 0.01 \\
& & $(0.061)$ & $(0.055)$ & $(0.056)$ \\
Ability - child & & $0.27^{* * *}$ & $0.14^{* * *}$ & $0.14^{* *}$ \\
& & $(0.061)$ & $(0.054)$ & $(0.055)$ \\
Yrs. schooling - mother & & 0.01 & -0.00 & -0.00 \\
& & $0.04^{* * *}$ & $0.03^{* * *}$ & $0.02^{* * *}$ \\
Yrs. schooling - child & & $(0.007)$ & $(0.006)$ & $(0.006)$ \\
& & & 0.01 & 0.00 \\
Net worth - mother & & & $(0.011)$ & $(0.011)$ \\
& & & -0.01 & -0.01 \\
Net worth - child & & & $(0.010)$ & $(0.010)$ \\
& & & & -0.00 \\
Number of children - mother & & & & $(0.013)$ \\
Number of children - child & & & & $-0.06 * * *$ \\
Control age at birth - mother & NO & NO & NO & YES \\
\hline Observations & 0.04 & 0.08 & 0.05 & $0.073)$ \\
\hline
\end{tabular}

Notes: Standard errors clustered at the mother level in parentheses. ${ }^{* * *} \mathrm{p}<0.01,{ }^{* *} \mathrm{p}<0.05,{ }^{*} \mathrm{p}<0.1$. In all columns, we use the same covariates as we do for the main results in Table 2, except that we also take the logarithm of maternal employment. 
Table C.10: Robustness: Rank-rank regressions

Dependent variable: Employment rank - child

\begin{tabular}{lcccc}
\hline Specification & $(1)$ & $(2)$ & $(3)$ & $(4)$ \\
\hline Employment rank - mother & $0.12^{* * *}$ & $0.08^{* * *}$ & $0.06^{* * *}$ & $0.06^{* * *}$ \\
& $(0.018)$ & $(0.018)$ & $(0.017)$ & $(0.017)$ \\
Ability - mother & & $0.04^{*}$ & 0.03 & 0.01 \\
& & $(0.020)$ & $(0.019)$ & $(0.018)$ \\
Ability - child & & $0.08^{* * *}$ & $0.05^{* * *}$ & $0.04^{* *}$ \\
& & $(0.019)$ & $(0.018)$ & $(0.018)$ \\
Yrs. schooling - mother & & 0.00 & 0.00 & 0.00 \\
& & $0.01^{* * *}$ & $0.01^{* * *}$ & $0.01^{* * *}$ \\
Yrs. schooling - child & & $(0.002)$ & $(0.002)$ & $(0.002)$ \\
& & & 0.01 & 0.00 \\
Net worth - mother & & & $(0.004)$ & $(0.004)$ \\
& & & $-0.01^{*}$ & $-0.01^{* *}$ \\
Net worth - child & & & $(0.005)$ & $(0.004)$ \\
Number of children - mother & & & & 0.00 \\
& & & & $(0.004)$ \\
Number of children - child & & & & $-0.03^{* * *}$ \\
Adjusted $R^{2}$ & & & & $(0.004)$ \\
\hline
\end{tabular}

Notes: Standard errors clustered at the mother level in parentheses. ${ }^{* * *} \mathrm{p}<0.01,{ }^{*} \mathrm{p}<0.05,{ }^{*} \mathrm{p}<0.1$. In all columns, we use the same covariates as we do for the main results in Table 2 . 
Table C.11: Robustness: Alternative measures of the permanent components

Dependent variable: Alternative permanent component employment - child $\left(\overline{l_{C i}}\right)$

\begin{tabular}{|c|c|c|c|c|}
\hline \multirow[b]{2}{*}{ Specification } & \multicolumn{2}{|c|}{ Simple averages } & \multicolumn{2}{|c|}{ Demographics } \\
\hline & (1) & (4) & (1) & (4) \\
\hline Employment - mother (averages) & $\begin{array}{c}0.21^{* * *} \\
(0.028)\end{array}$ & $\begin{array}{c}0.12^{* * *} \\
(0.027)\end{array}$ & & \\
\hline Employment - mother (demographics) & & & $\begin{array}{c}0.21^{* * *} \\
(0.029)\end{array}$ & $\begin{array}{c}0.12^{* * *} \\
(0.028)\end{array}$ \\
\hline Controls & $\mathrm{NO}$ & YES & $\mathrm{NO}$ & YES \\
\hline Observations & 2,339 & 1,969 & 2,245 & 1,877 \\
\hline Adjusted $R^{2}$ & 0.04 & 0.08 & 0.03 & 0.04 \\
\hline
\end{tabular}

Notes: Standard errors clustered at the mother level in parentheses. ${ }^{* * *} \mathrm{p}<0.01,{ }^{* *} \mathrm{p}<0.05,{ }^{*} \mathrm{p}<0.1$. In columns one and two, we use simple averages for $l_{C i}$ and $l_{M i}$. In columns three and four, we add to the standard estimation of the permanent components demographic events as additional controls.

Table C.12: Robustness: Alternative survey questions for employment status of children and mothers

Dependent variable: Alternative data measure of employment - child $\left(\widehat{l_{C i}}\right)$

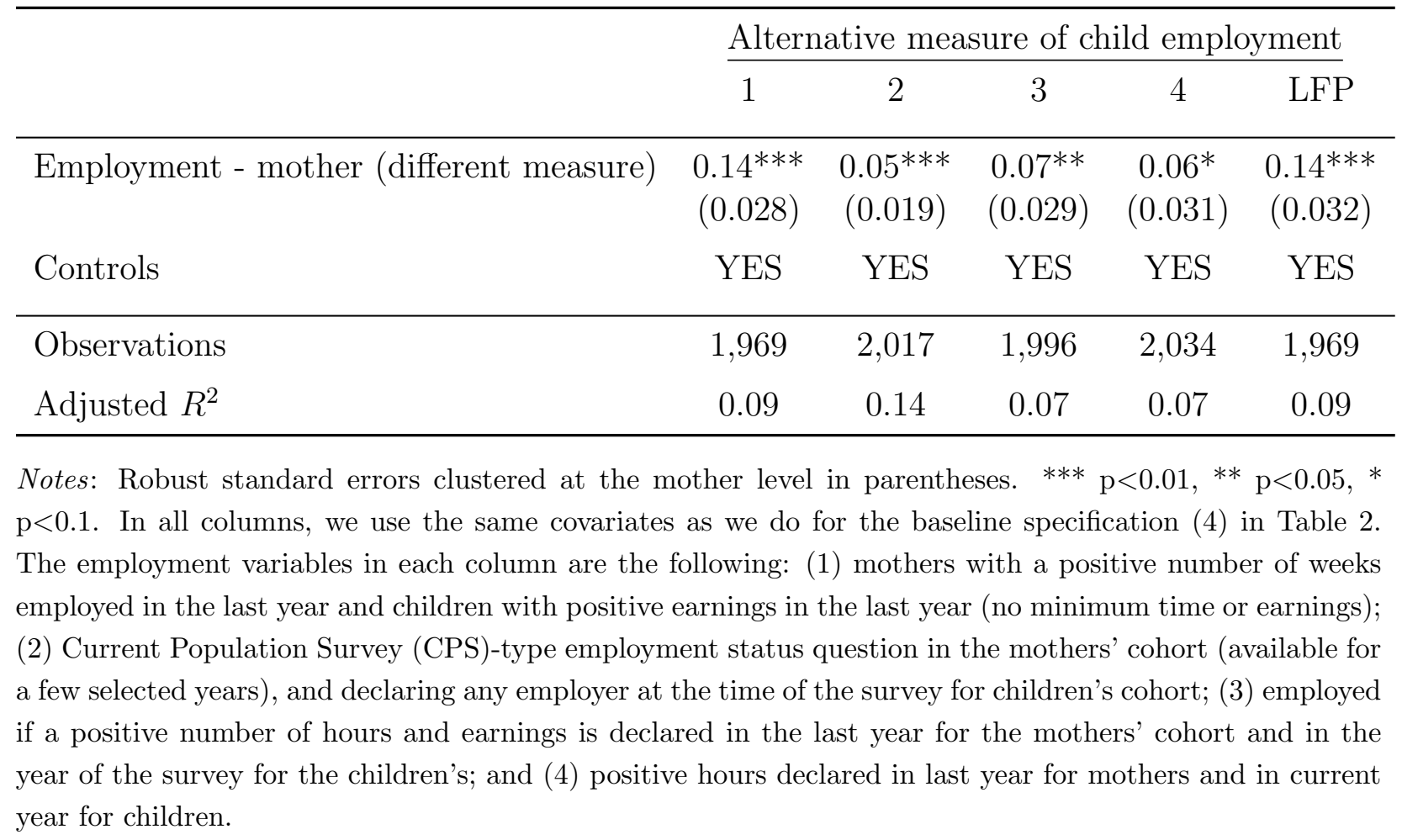


Table C.13: Heterogeneity: Intergenerational correlation of employment status by (i) family income (quintiles) and (ii) mother's education level

Dependent variable: Employment - child $\left(l_{C i}\right)$

\begin{tabular}{lccc}
\hline & Baseline & Family income & Maternal education \\
\hline Employment - mother & $0.12^{* * *}$ & $0.18^{* * *}$ & $0.21^{* * *}$ \\
Employment - mother $\times$ Quintile 2 & $(0.027)$ & $(0.059)$ & $(0.076)$ \\
Employment - mother $\times$ Quintile 3 & -0.08 & \\
Employment - mother $\times$ Quintile 4 & & $-0.095)$ & \\
Employment - mother $\times$ Quintile 5 & & $(0.086)$ & \\
Employment - mother $\times$ Complete high-school & & $-0.18^{* *}$ & \\
Employment - mother $\times$ Incomplete college & & $-0.081)$ & -0.09 \\
Employment Mother $\times$ Complete college & & & $(0.074)$ \\
Controls & & & $-0.24^{* * *}$ \\
\hline Observations & & & $(0.091)$ \\
Adjusted $R^{2}$ & & & -0.09 \\
\hline
\end{tabular}

Notes: Standard errors clustered at the mother level in parentheses. ${ }^{* * *} \mathrm{p}<0.01,{ }^{* *} \mathrm{p}<0.05,{ }^{*} \mathrm{p}<0.1$. Quintiles of family income correspond to the quintile of family income observed most often. The maternal education is the maximum attained education level. In all columns, we use the same covariates as we use in the baseline specification (4) in Table 2 ability, years of schooling, net worth, and number of children for mothers and children, as well as mother's age at birth. 
Table C.14: Descriptive statistics for mothers' disutility from work by terciles

\begin{tabular}{lccccc}
\hline & $\min$ & $\max$ & $\operatorname{mean}$ & $\mathrm{sd}$ & Observations \\
\hline Low disutility from work & -1.500 & -0.750 & -0.960 & 0.189 & 831 \\
Medium disutility from work & -0.667 & -0.375 & -0.518 & 0.099 & 804 \\
High disutility from work & -0.333 & 1.250 & 0.037 & 0.308 & 798 \\
\hline All observations & -1.500 & 1.250 & -0.487 & 0.462 & 2433 \\
\hline
\end{tabular}

Notes: Disutility from work computed from questions on women's roles: (i) Women's place is in the home, not in the office or shop, and (ii) Women are much happier if they stay at home and take care of the children. These questions are included only in survey years 1979, 1982, 1987, and 2004. We assign the values (a) strongly agree 1.5 , (b) agree 0.5 , (c) disagree -0.5 , and (d) strongly disagree -1.5 . We average across questions and across years.

Table C.15: Employment - proportion of periods employed in the lifetime - of children and mothers, by terciles of mothers' employment and disutility from work

\begin{tabular}{lcc}
\hline Employment & Children & Mothers \\
\hline Mother's employment & & \\
$1^{\text {st }}$ Tercile & 0.77 & 0.34 \\
$2^{\text {nd }}$ Tercile & 0.86 & 0.80 \\
$3^{\text {rd }}$ Tercile & 0.89 & 0.99 \\
\hline Mother's disutility from work & & \\
$1^{\text {st }}$ Tercile & 0.84 & 0.78 \\
$2^{\text {nd }}$ Tercile & 0.85 & 0.74 \\
$3^{\text {rd }}$ Tercile & 0.83 & 0.60 \\
\hline
\end{tabular}

Notes: Employment of mother and child correspond to the averages across years and individuals. Disutility from work: (i) Women's place is in the home, not in the office or shop, and (ii) Women are much happier if they stay at home and take care of the children. We assign the values (a) strongly agree 1.5, (b) agree 0.5, (c) disagree -0.5 , and (d) strongly disagree -1.5. We average across questions and across years. 
Table C.16: Direct preference transmission vs. role model: Direct preference transmission proxied by employment status based on periods of non-cohabitation

Dependent variable: Employment - child $\left(l_{C i}\right)$

\begin{tabular}{lccc}
\hline Specification & Baseline & Maternal preferences & Full \\
\hline Employment - mother & $\begin{array}{c}0.16^{* * *} \\
(0.037)\end{array}$ & & $0.14^{* * *}$ \\
& & $0.07^{* * *}$ & $(0.041)$ \\
Employment - mother when... & & $(0.027)$ & 0.02 \\
...not cohabiting with child & & YES & YES \\
Controls & YES & 1,123 & 1,123 \\
\hline Observations & 1,123 & 0.10 & 0.11 \\
Adjusted $R^{2}$ & 0.11 & & $0.030)$ \\
\hline
\end{tabular}

Notes: Standard errors clustered at the mother level in parentheses. ${ }^{* * *} \mathrm{p}<0.01,{ }^{* *} \mathrm{p}<0.05,{ }^{*} \mathrm{p}<0.1$. We use the same covariates as we do in the baseline specification (4) in Table 2 ability, years of schooling, net worth, and number of children for mothers and children, as well as mother's age at birth. Periods of non-cohabitation are specific for each child-mother pair. Only pairs with both periods of cohabitation and non-cohabitation are included. 


\section{C.2 Additional figures}

Figure C.1: Visual example of a mother-child pair

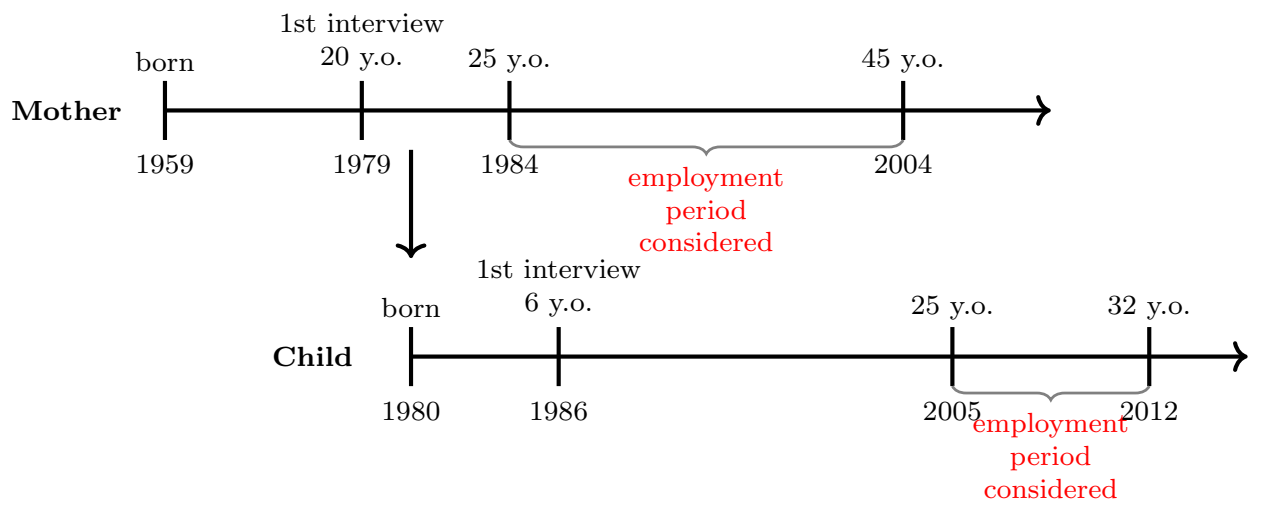

Figure C.2: Number of interviews of mothers (left) and children (right)
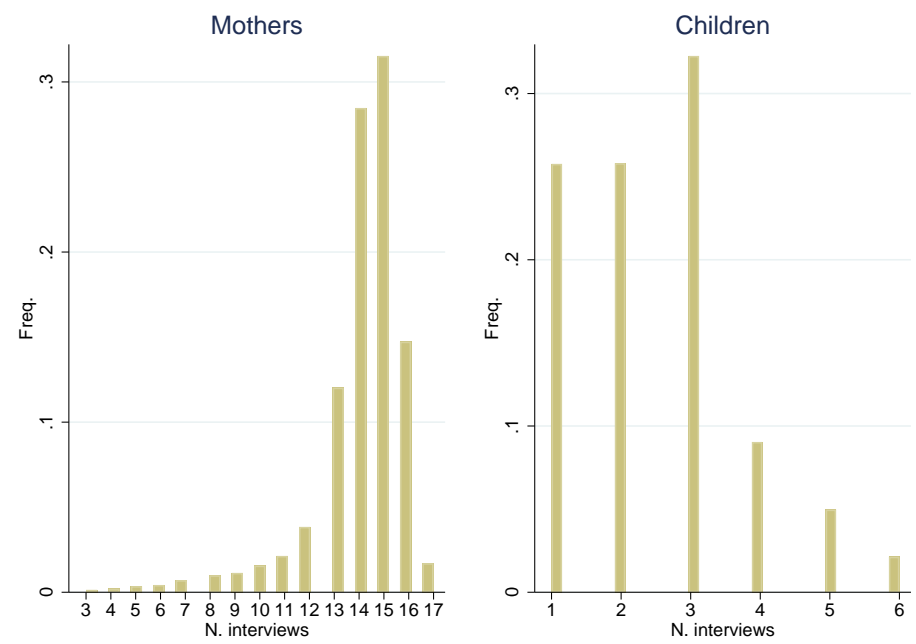
Figure C.3: Age of mothers at birth of child
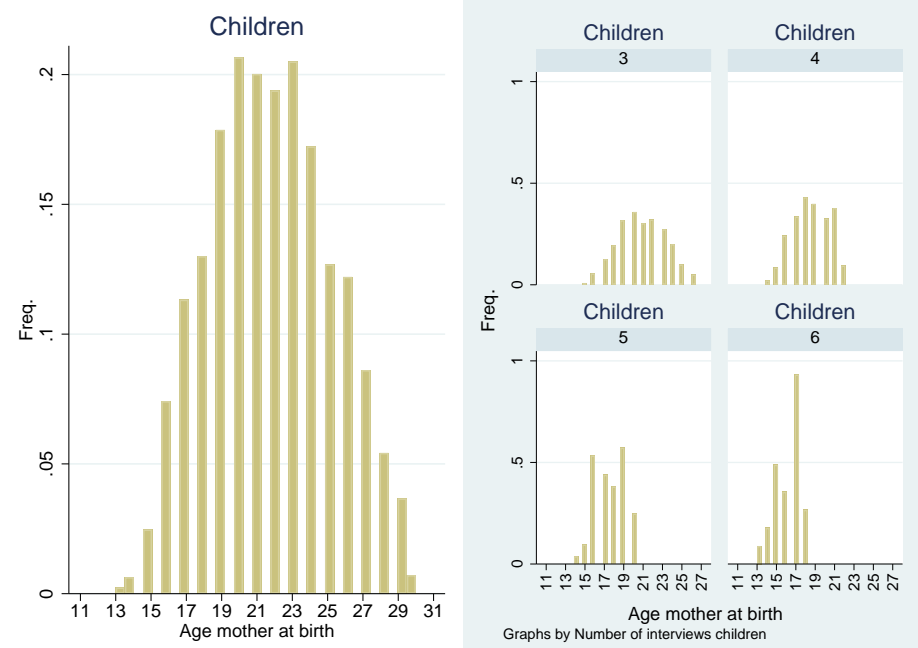

Figure C.4: Employment-age profiles of mothers (left) and children (right)
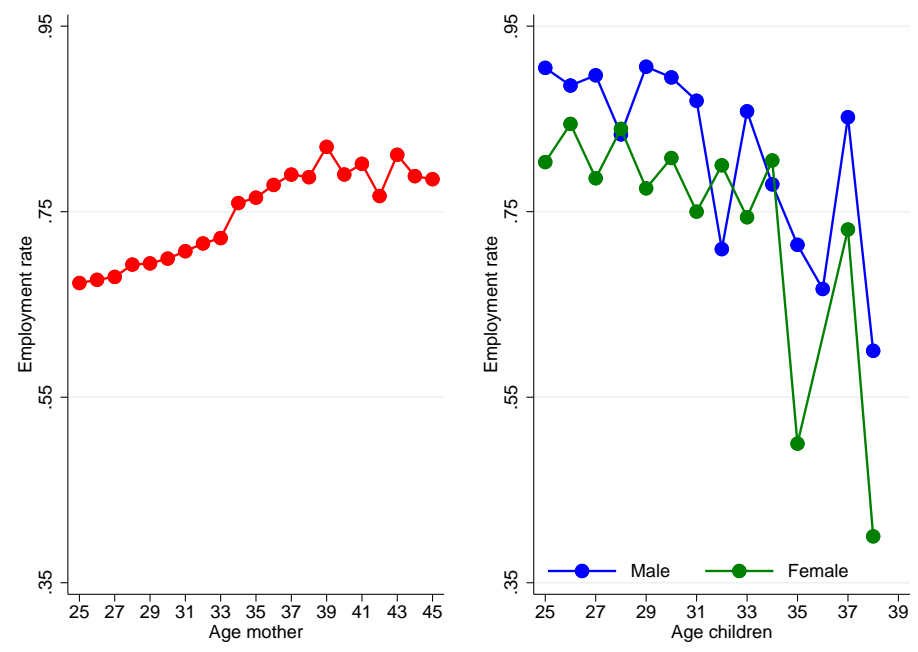
Figure C.5: Intergenerational correlation of employment status by mother's income (left) and education (right) for sons and daughters
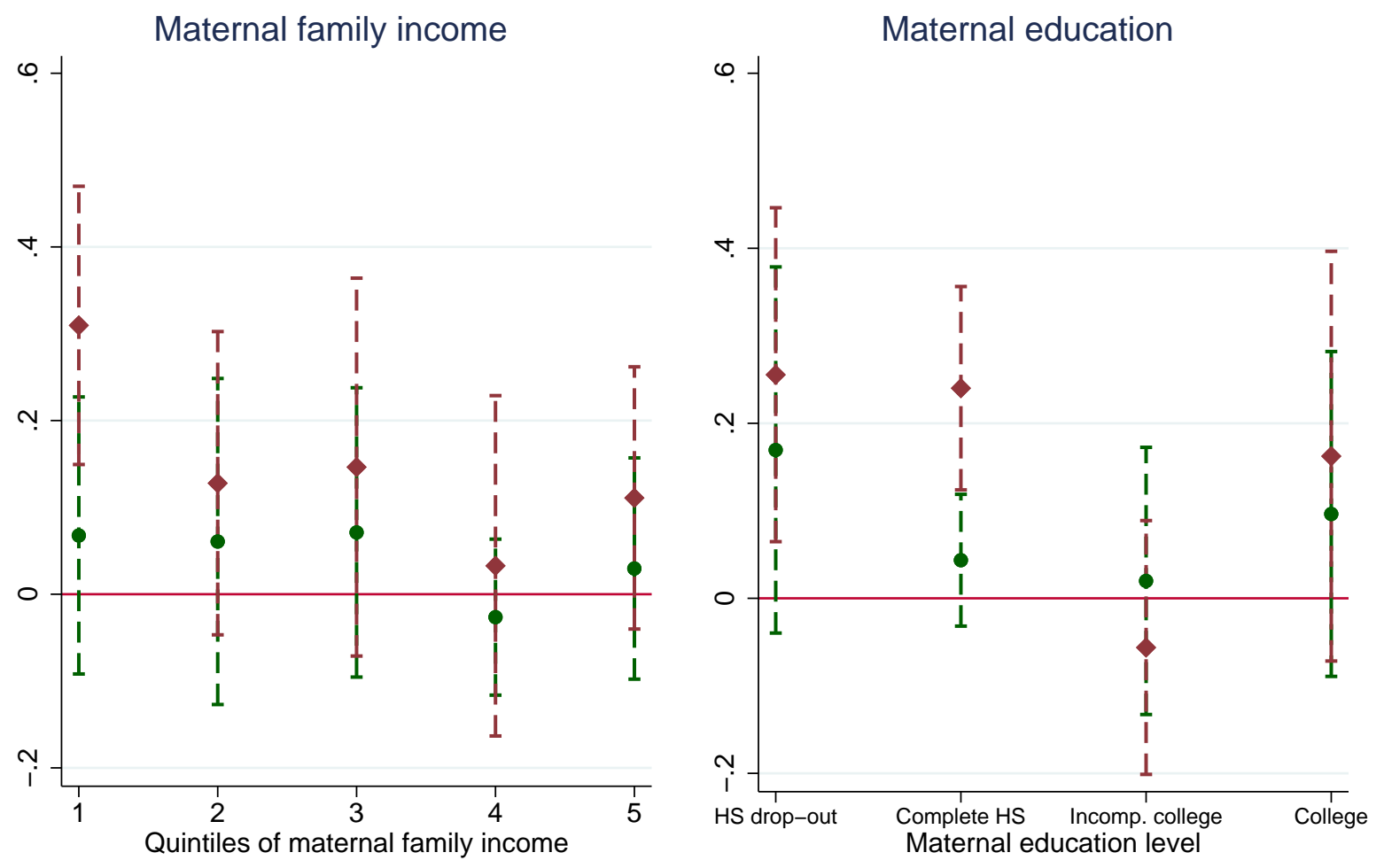

- Sons Daughters

Note: The dependent variable is the permanent component of the natural logarithm of employment status of the children. Standard errors are clustered by mother ID. Mother's position in the income distribution is attributed according to the quintile observed during the maximum number of waves. The education level of mothers is the maximum attained. 
Figure C.6: Distribution of maternal disutility from work

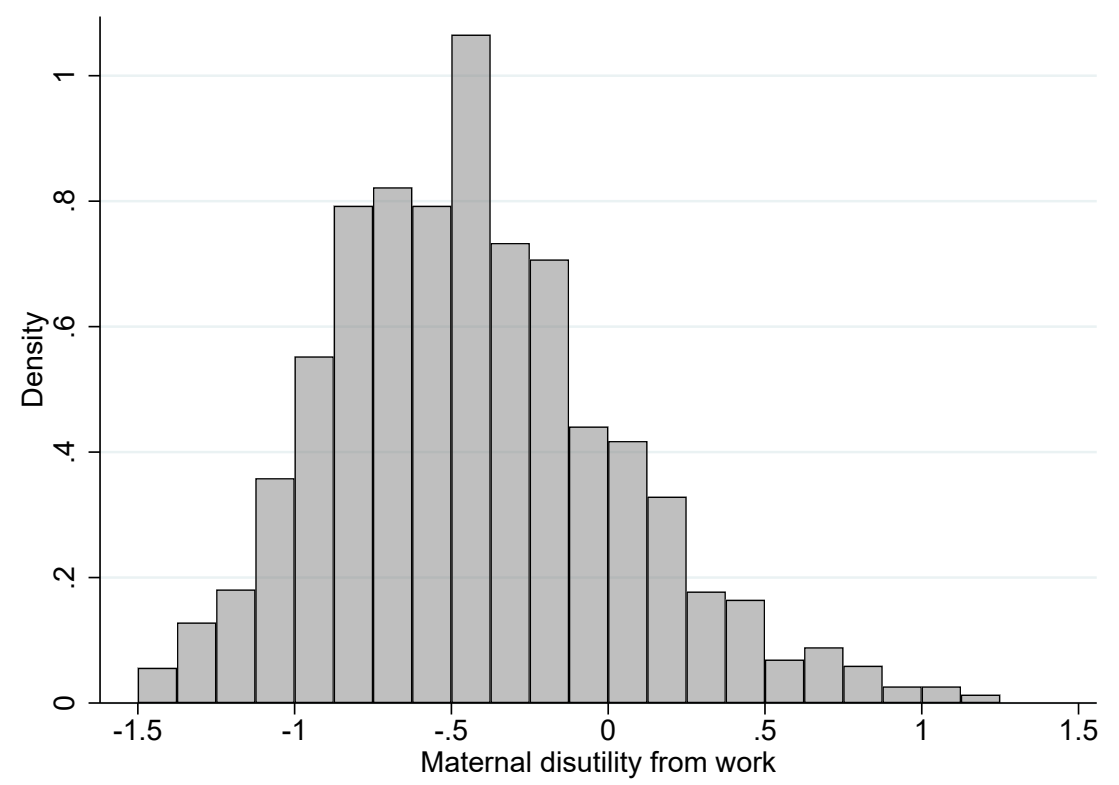

Notes: Disutility from work computed from questions on women's roles: (i) Women's place is in the home, not in the office or shop, and (ii) Women are much happier if they stay at home and take care of the children. These questions are included only in survey years 1979, 1982, 1987, and 2004. We assign the values (a) strongly agree 1.5 , (b) agree 0.5 , (c) disagree -0.5 , and (d) strongly disagree -1.5 . We average across questions and across years. 